\title{
Spatially-resolved correlative microscopy and microbial identification reveals dynamic depth- and mineral-dependent anabolic activity in salt marsh sediment
}

\author{
Jeffrey Marlow ${ }^{1, \#}$, Rachel Spietz ${ }^{2}$, Keun-Young Kim³ ${ }^{3}$, Mark Ellisman ${ }^{3,4}$, Peter Girguis ${ }^{1}$, and \\ Roland Hatzenpichler ${ }^{2, *}$ \\ 1: Department of Organismic and Evolutionary Biology, Harvard University, 16 Divinity Ave., Cambridge, MA, \\ 02138, USA; 2: Department of Chemistry and Biochemistry, Thermal Biology Institute, and Center for Biofilm \\ Engineering, Montana State University, Bozeman, MT, 59717, USA; 3: Department of Neurosciences, University of \\ California at San Diego School of Medicine and National Center for Microscopy and Imaging Research, University \\ of California, San Diego, La Jolla, CA, 92093, USA; 4:Department of Pharmacology, University of California, San \\ Diego, La Jolla, CA, 92161, USA. \\ \# Current address: Boston University Dept. of Biology, 5 Cummington Mall, Room 101, Boston, MA, 02215, USA \\ * To whom correspondence should be addressed: \\ 111 Chemistry \& Biochemistry Bldg., Montana State University, PO Box 173400, Bozeman, MT-59717 \\ Phone: 406-994-5469; Fax: 406-994-5407; Email: roland.hatzenpichler@montana.edu.
}

Running Title: Correlative microscopy in salt marsh sediment 


\section{Statement of Significance}

Microscale spatial relationships dictate critical aspects of a microbiome's inner workings and emergent properties, such as evolutionary pathways, niche development, and community

4 structure and function. However, many commonly used methods in microbial ecology neglect this

5 parameter - obscuring important microbe-microbe and microbe-mineral interactions - and instead

6 employ bulk-scale methodologies that are incapable of resolving these intricate relationships.

This benchmark study presents a compelling new approach for exploring the anabolic activity of a complex microbial community by mapping the precise spatial configuration of

9 anabolically active organisms within mineralogically heterogeneous sediment through in situ

10 incubation, resin embedding, and correlative fluorescence and electron microscopy. In parallel,

11 active organisms were identified through fluorescence-activated cell sorting and 16S rRNA gene

12 sequencing, enabling a powerful interpretive framework connecting location, identity, activity, and

13 putative biogeochemical roles of microbial community members.

14 We deploy this novel approach in salt marsh sediment, revealing quantitative insights into

15 the fundamental principles that govern the structure and function of sediment-hosted microbial

16 communities. In particular, at different sediment horizons, we observed striking changes in the

17 proportion of anabolically active cells, the identities of the most prominent active community

18 members, and the nature of microbe-mineral affiliations. Improved approaches for understanding

19 microscale ecosystems in a new light, such as those presented here, reveal environmental

20 parameters that promote or constrain metabolic activity and clarify the impact that microbial

21 communities have on our world.

22 Abstract 
Coastal salt marshes are key sites of biogeochemical cycling and ideal systems in which to

24 investigate the community structure of complex microbial communities. Here, we clarify

25 structural-functional relationships among microorganisms and their mineralogical environment,

26 revealing previously undescribed metabolic activity patterns and precise spatial arrangements

27 within salt marsh sediment. Following 3.7-day in situ incubations with a non-canonical amino acid

28 that was incorporated into new biomass, samples were embedded and analyzed by correlative

29 fluorescence and electron microscopy to map the microscale arrangements of anabolically active

30 and inactive organisms alongside mineral grains. Parallel sediment samples were examined by

31 fluorescence-activated cell sorting and 16S rRNA gene sequencing to link anabolic activity to

32 taxonomic identity. Both approaches demonstrated a rapid decline in the proportion of anabolically

33 active cells with depth into salt marsh sediment, from $\sim 60 \%$ in the top cm to $10-25 \%$ between $2-7$

$34 \mathrm{~cm}$. From the top to the bottom, the most prominent active community members shifted from sulfur

35 cycling phototrophic consortia, to sulfate-reducing bacteria likely oxidizing organic compounds,

36 to fermentative lineages. Correlative microscopy revealed more abundant (and more anabolically

37 active) organisms around non-quartz minerals including rutile, orthoclase, and plagioclase.

38 Microbe-mineral relationships appear to be dynamic and context-dependent arbiters of

39 biogeochemical cycling.

\section{Introduction}

41 Salt marshes are vibrant microbial habitats that play important roles in the biogeochemical

42 cycling of intertidal ecosystems (Tobias and Neubauer, 2019). The confluence of high organic

43 input and seawater-derived sulfate fuel a wide range of carbon, nitrogen, phosphorous, and sulfur

44 transformations over compressed spatial scales, leading to abundant, redox-specific niches and

45 microbial communities with high phylogenetic diversity (Lozupone and Knight, 2007; Bowen et 
al., 2012). Because of this, salt marshes represent ideal sites to explore the intricacies of microbial community structure from the microscale to the ecosystem scale.

Within complex microbial communities, spatial relationships are increasingly seen as

49 central determinants of key ecological parameters. In salt marshes, metabolic activity within

50 specific sediment horizons ultimately shapes emergent properties such as carbon sequestration or

51 greenhouse gas emissions (Abdul-Aziz et al., 2018; LaRowe et al., 2020). More generally,

52 microbe-microbe and microbe-mineral interactions establish evolutionary trajectories (Cordero et

53 al., 2012; Andersen et al., 2015), niche development (Morton et al., 2017), and community

54 structure, function, and stability (Boetius et al., 2000; Wright et al., 2012; Coyte et al., 2015).

55 Further, inter-organism arrangements govern chemical communication (West et al., 2007),

56 metabolite exchange (Romine et al., 2017), and competition for resources (Mitri et al., 2016).

57 Nonetheless, these critical spatial relationships are neglected by the most commonly used methods

58 in microbial ecology, such as bulk meta-omics and geochemical approaches. As a result, important

59 metabolic activities may be obscured, including inter-species nutrient cycling (Wilbanks et al.,

60 2014; Cordero and Datta, 2016) and electron transfer to (Lovley and Phillips, 1988; Myers and

61 Nealson, 1988) or from (Shelobolina et al., 2012) specific minerals.

Recent efforts have made progress in analyzing microbial communities at the microscale.

63 nanoscale secondary ion mass spectrometry (nanoSIMS) coupled with stable isotope probing (SIP)

64 and fluorescence in situ hybridization (FISH) can resolve anabolic patterns and taxonomically

65 identify individual cells. However, this method typically separates microbial assemblages from

66 their broader environmental context (McGlynn et al., 2015; Musat et al., 2016; Gyngard and

67 Steinhauser, 2019). By combining energy dispersive x-ray spectroscopy (EDS) with x-ray

68 computed tomography images, Hapca et al. extended chemical analyses into a third dimension 
69 with resin-embedded soil, but no cellular information was attained (Hapca et al., 2015). Correlative

70 imaging with nanoSIMS and electron and fluorescence microscopy enabled Schlüter et al. to

71 pinpoint the position of a subset of the microbial community in relation to leaf fragments, but

72 metabolic activity and microbial identities were not considered (Schlüter et al., 2018). A promising

73 addition to this emerging field is SIP combined with non-destructive Confocal Raman

74 microspectroscopy, which was recently used to measure the in situ activity and substrate uptake

75 of microbes in transparent soil microcosms (Sharma et al., In Press).

The work presented here advances this line of microbial ecology research. The methods herein not only preserve spatial arrangements and link cell positions to mineralogy through correlative microscopy, but also establish the presence, location, and mineralogical associations of anabolically active cells. Anabolic activity was assessed with bioorthogonal non-canonical amino acid tagging (BONCAT), a next-generation physiology approach (Hatzenpichler et al., 2020) that uses substrate analog probing to visualize protein synthesis in active cells. A non-canonical amino acid, such as $L$-homopropargylglycine (HPG) or $L$-azidohomoalanine (AHA), is incorporated into

83 growing peptides by native methionyl-tRNA synthetases. Subsequent azide-alkyne click chemistry

84 allows fluorescent detection of newly synthesized proteins (Sletten and Bertozzi, 2009). BONCAT

85 was initially developed in neuron (Dieterich et al., 2006), eukaryote (Hinz et al., 2011), and

86 cultured bacteria (Hatzenpichler et al., 2014) systems; more recently, it was optimized for

87 environmental microbial communities and shown to have no measurable effect on community 88 composition or metabolic activity (Hatzenpichler et al., 2014, 2016). The approach has been 89 proven effective in a diverse range of bacterial and archaeal cultures (Hatzenpichler et al., 2014; 90 Hatzenpichler and Orphan, 2015); ocean water (Samo et al., 2014; Leizeaga et al., 2017; Sebastián 91 et al., 2019), marine sediment (Hatzenpichler et al., 2016), hot spring (Reichart et al., 2020), and 
92 soil microbiomes (Couradeau et al., 2019); as well as marine viruses and bacteriophages (Pasulka

93 et al., 2018). BONCAT appears to be a taxonomically agnostic measure of anabolic activity that

94 correlates well with other metrics of activity (Bagert et al., 2014; Hatzenpichler et al., 2014, 2020)

95 with only small effects on metabolism (Steward et al., 2020) and protein chemistry (Bagert et al.,

96 2014; Lehner et al., 2017).

97 In this study, we mapped the anabolic activity of individual microorganisms in sediments

98 from Little Sippewissett Salt Marsh (LSSM) in Falmouth, MA. In the LSSM, terrestrial freshwater

99 runoff, seawater, high organic input, and abundant light and chemical energy leads to dramatic

100 redox stratifications within the top few centimeters of sediment and a wide range of metabolic

101 niches (Armitage et al., 2012; Wilbanks et al., 2014, 2017; Larsen et al., 2015). Using purpose-

102 built equipment, a series of sediment cores were incubated with HPG in situ for 3.7 days. One set

103 of cores was used for correlative microscopy; samples were embedded in resin to maintain precise

104 spatial arrangements, sectioned, stained, and analyzed using fluorescence and electron microscopy

105 to map active and inactive biomass as well as identifiable mineral grains. A parallel set of cores

106 was processed for horizon-specific fluorescence activated cell sorting (FACS) and 16S rRNA gene

107 amplicon sequencing. With this novel approach, we mapped active and inactive organisms in their

108 native microscale configuration and identified the active and inactive microbial communities in

109 adjacent sediment horizons.

110 Our results indicate that the proportion of anabolically active organisms decreased

111 dramatically below the photic zone, and that mineralogy likely has an impact on the relative

112 abundance and anabolic activity of mineral grain-associated organisms. High-throughput 16S

113 rRNA gene sequencing of active and inactive microbial communities in adjacent sediment cores

114 revealed a continuous progression of community structure with depth, oriented around shifting 
115 metabolisms of photosynthesis, sulfur cycling, and fermentation. Notably, with correlative

116 fluorescence and electron microscopy, we observed differential cell association with distinct

117 mineral types and a greater proportion of organisms inside mineral grains in lower $(6-7 \mathrm{~cm})$

118 sediment horizons compared with shallower zones. While the full potential of microbiome

119 mapping remains to be realized, this benchmarking study unveils a new experimental approach to

120 a) evaluate how metabolic activity relates to microscale environmental factors, and b) develop

121 testable hypotheses regarding metabolic interactions among members of complex microbial

122 communities.

123 Results \& Discussion

124 This study reveals how microbial presence and anabolic activity relate to mineralogical

125 distributions at the microscale with a new level of realism in salt marsh sediment. Correlative

126 microscopy analyses at three distinct horizons revealed changes in organism abundance from

$1271.95 \times 10^{9} \mathrm{~cm}^{-3}$ at $7.6 \mathrm{~mm}$ depth to $2.86 \times 10^{9} \mathrm{~cm}^{-3}$ at $12 \mathrm{~mm}$ depth and $6.85 \times 10^{8} \mathrm{~cm}^{-3}$ at $60.7 \mathrm{~mm}$

128 depth. Moving downward along these three horizons, the proportion of anabolically active

129 organisms decreased from $51.3 \%(7.6 \mathrm{~mm})$ to $22.3 \%(12 \mathrm{~mm})$ to $12.1 \%(60.7 \mathrm{~mm})$, a trend that

130 correlated well with BONCAT-FACS data $\left(\mathrm{R}^{2}=0.99\right.$; Table 1$)$.

131 At each microscopy horizon, the mineralogical identities of individual grains were assessed

132 in order to determine whether different mineral types corresponded with notable differences in

133 organism abundance, configuration, or anabolic activity. The majority of all detected grains were

134 quartz $\left(\mathrm{SiO}_{2}\right)$, while albite $\left(\mathrm{NaAlSi}_{3} \mathrm{O}_{8}\right)$, orthoclase $\left(\mathrm{KAlSi}_{3} \mathrm{O}_{8}\right)$, rutile $\left(\mathrm{TiO}_{2}\right)$, plagioclase (a solid

135 solution range from $\mathrm{NaAlSi}_{3} \mathrm{O}_{8}$ to $\mathrm{CaAl}_{2} \mathrm{Si}_{2} \mathrm{O}_{8}$ ), and $\mathrm{Ca} / \mathrm{K} / \mathrm{Mg} / \mathrm{Fe}$ silicate grains of indeterminant

136 mineralogy were also detected. Like the vocabulary used to describe microbe-microbe interactions,

137 microbe-mineral interactions can be harmful, neutral, or beneficial for the organism. Microbial 
138 sorption to quartz grains has been demonstrated, but repellant electrical charges make the 139 interaction less favorable than those with other mineral types (Mills et al., 1994; Gong et al., 2018).

140 The best-studied beneficial interactions are the microbial reduction of iron or manganese oxides

141 (Thamdrup, 2000), which enables bacteria to off-load reducing power, altering mineral structure

142 and chemistry in the process (Kawano and Tomita, 2002; Welch and Banfield, 2002). A number

143 of factors influence the nature of these interactions, including accessible surface area, mineral

144 lattice structure, co-occurrence of organic matter, and other environmental conditions such as

145 temperature and $\mathrm{pH}$ (Dong et al., 2009). Beyond iron and manganese, microbes have been shown

146 to associate with other cations, acquiring potassium from silicates (Valsami-Jones et al., 1998),

147 releasing organic ligands that adhere to aluminum (Rogers and Bennett, 2004), and using reducing

148 power from photo-catalytically activated titanium oxide (Lu et al., 2012).

149 Top horizon BONCAT-FACS \& Correlative Microscopy

150 Many previous studies have elucidated key aspects of the LSSM microbiological system 151 and its role in biogeochemical cycling (Seitz et al., 1993; Shapiro et al., 2011; Armitage et al.,

152 2012; Bowen et al., 2013; Peng et al., 2013; Wilbanks et al., 2014, 2017; Larsen et al., 2015;

153 Mackey et al., 2017; Angell et al., 2018); we leverage this heritage to infer physiological traits

154 based on the 16S rRNA gene data we collected. (Please see Supporting Information Dataset 1 for

155 sequence data and relative abundances of assigned lineages for bulk, active, and inactive samples 156 across all horizons.)

157 The top ten millimeters of LSSM sediment exhibit dramatic redox gradients as oxygen 158 concentrations fall below detection by $5 \mathrm{~mm}$, sulfide rises from 0 to between $0.5-1.5 \mathrm{mM}$, and $\mathrm{pH}$ 159 fluctuates between $~ 7.0-7.3$ at night and $\sim 6.0-7.0$ during the day (Armitage et al., 2012; Larsen et 160 al., 2015; Salman et al., 2015). The microbial community was dominated by the phyla 
161 Proteobacteria (48\% relative abundance) and Bacteroidetes (30\%), whose metabolically diverse

162 members are indicative of a range of redox conditions and substantial heterotrophic cycling in the

163 upper sediment layer (Spain et al., 2009; Gómez-Pereira et al., 2012). Thiohalocapsa was the most

164 abundant genus-level lineage recovered, accounting for $14.4 \%$ of all sequences; Desulfobulbaceae

165 was the next most abundant genus, with two unidentified lineages representing $3.8 \%$ and $2.7 \%$ of

166 all sequences. The prevalence of these purple sulfur bacteria and sulfate-reducing bacteria is

167 reflective of the abundant "pink berries" found at the sediment surface (Fig. S1) (Seitz et al., 1993;

168 Wilbanks et al., 2014). Among organisms putatively involved with sulfur-cycling consortia, we

169 observed a more diverse distribution of sulfate-reducing bacteria lineages (65 genus-level

170 Desulfobacterales ASVs) and a more streamlined set of purple sulfur bacteria with a single

171 dominant representative (19 genus-level Chromatiales ASVs, with Thiohalocapsa accounting for

$17283 \%$ of the recovered sequences).

173 During the 3.7-day incubation period, the majority of organisms detected in this sediment

174 zone demonstrated anabolic activity (Table 1). Sequencing of active and inactive communities in

175 the $0-10 \mathrm{~mm}$ range revealed eight lineages representing $>1 \%$ of the overall relative abundance that

176 were significantly more abundant in the anabolically active subset (Dataset 1). Of these, six were

177 putative members of the pink berry consortia (Chromatiales or Desulfobacterales orders), one was

178 a photoheterotroph that may encode multiple light-harvesting complexes (Halieaceae, (Spring et

179 al., 2015)), and one was a representative of the metabolically diverse Rhodobacteraceae family

180 (Pujalte et al., 2014; Pohlner et al., 2019). Many of the other abundant inactive lineages - including

181 three putative sulfate reducers and three putative purple sulfur bacteria - were among the most

182 abundant ASVs in both the active and inactive fractions (Dataset 1). This overlap may indicate

183 stochastic activity of particular consortia or a metabolic dependence upon physicochemical traits 
184 on a sub-cm scale, such as pore connectivity or identity of neighboring organisms. Alternatively,

185 our conservative gating approach may have captured some active cells with low fluorescence in

186 the inactive gate (Fig S2). Among the inactive microorganisms, two Rhizobiaceae lineages

187 constituted a combined $11.8 \%$ of the sequenced fraction. This family of Alphaproteobacteria is

188 typically associated with actively growing plants (Spaink et al., 2012); their anabolic quiescence

189 could be attributable to displacement from spartina grass roots surrounding the sample site.

191 sequenced horizon, at a depth of $7.6 \mathrm{~mm}$ (Fig. 1). In the analysis area, 15 of the 20 mineral grains

192 were quartz $\left(\mathrm{SiO}_{2}\right)$, while albite $\left(\mathrm{NaAlSi}_{3} \mathrm{O}_{8}\right)$, orthoclase $\left(\mathrm{KAlSi}_{3} \mathrm{O}_{8}\right)$, rutile $\left(\mathrm{TiO}_{2}\right)$, plagioclase $(\mathrm{a}$

193 solid solution range from $\mathrm{NaAlSi}_{3} \mathrm{O}_{8}$ to $\mathrm{CaAl}_{2} \mathrm{Si}_{2} \mathrm{O}_{8}$ ), and $\mathrm{Ca} / \mathrm{K} / \mathrm{Mg} / \mathrm{Fe}$ silicate grains of

194 indeterminant mineralogy were also observed. $73.4 \%$ of cells were located within $70 \mu \mathrm{m}$ or found

195 inside of quartz grains. When cell biomass abundances were normalized by proxies for mineral

196 surface area and volume, non-quartz grains exhibited a higher organism density (Table 2).

198 were found inside, frequently along fractures or pores visible by SEM. The most biomass-rich

199 zone was the "surface-associated" $0-5 \mu \mathrm{m}$ bin, where $27.8 \%$ of cells were found. Beyond $10 \mu \mathrm{m}$,

200 cell abundance decreased markedly, demonstrating a strong preference for grain interface zones

201 and reflecting the removal of suspended biomass when the original porewater was replaced with

202 filtered HPG solution prior to incubation. These spatial trends were broadly consistent across

203 different mineral types (Fig. 2), though the greatest proportion (39\%) of orthoclase-associated cells

204 were not in the "surface-associated" 0-5 $\mu \mathrm{m}$ bin, but in the 5-10 $\mu \mathrm{m}$ zone. We also found that the

205 degree of anabolic activity was higher around non-quartz minerals when compared with quartz-

206 associated cells (Table 2). Although low abundances of these mineral types make generalizations 
207 difficult, it is possible that metal cations in the mineral structures facilitate a wider range of

208 metabolic reactions than the more chemically inert quartz (Shi et al., 2016). The electrical semi-

209 conductivity of titanium oxide can promote extracellular electron transfer (Zhou et al., 2018) and,

210 via photo-catalysis, stimulate the growth of non-phototrophic microbes (Lu et al., 2012); these

211 mechanisms may account for the elevated proportion (78\%, compared with a mean of $51.7 \%$ for

212 this horizon) of active cells associated with the exterior of the titanium oxide rutile grain.

213 Second horizon BONCAT-FACS \& Correlative Microscopy

214 Between 10-20 mm of sediment depth, oxygen is absent and $\mathrm{pH}$ remains largely consistent

215 within a $\sim 0.3$ unit range, but sulfide concentrations exhibit substantial (up to $\sim 500 \mu \mathrm{M}$ ) fluctuations

216 upward or downward, with no clear pattern based on diurnal cycle timing (Larsen et al., 2015;

217 Salman et al., 2015). Under these more energetically constrained conditions, 16S rRNA gene reads

218 were dominated by Proteobacteria (40\%), Cyanobacteria (26\%), and Bacteroidetes (13\%) phyla

219 suggestive of recycling of plant material as well as vibrant nitrogen and sulfur cycling processes.

220 Among the ASVs representing more than 1\% of relative abundance, one ASV most similar to

221 mitochondria (2.8\%) and eight cyanobacterial chloroplast-like sequences (summing to $16.7 \%$ )

222 likely reflect burial and degradation of animal and photosynthetic biomass. Primers used in this

223 study can amplify some eukaryotic sequences (Parada et al., 2016), but the short sequence length

224 precludes accurate taxonomic assignment to eukaryotic chloroplast or mitochondria. Interestingly,

225 these constituents were seemingly selected against during the cell extraction and sorting process,

226 as their relative abundance in the active and inactive fractions were all well below $1 \%$.

227 Mitochondrial ASVs accounted for $0.12 \%$ and $0.01 \%$ of sequences in active and inactive fractions,

228 respectively; chloroplast ASVs were $0 \%$ and $0.01 \%$, respectively. 
The contribution of burial and degradation is consistent with the lower proportion of active

230 organisms observed by both microscopy (22.3\%) and cell sorting data (22.4\%). Sulfate-reducing

231 deltaproteobacterial clades (particularly those within the Desulfobacterales family) as well as the

232 environmentally wide-ranging betaproteobacterial family Burkholderiaceae, were more prevalent

233 among active organisms. Sulfate-reducing bacteria represented five of the seven ASVs that were

234 significantly enriched in the active fraction and accounted for at least $1 \%$ of the overall relative

235 abundance, indicating a vibrant sulfur cycle likely fueled by organic carbon degradation.

236 Rhizobiales and Chromatiaceae were more abundant in the inactive fraction, suggesting that

237 potentially critical environmental factors like viable plant cells and sunlight, respectively, were

238 not abundantly available. Nonetheless, one Chromatiaceae ASV (of the Halochromatium genus)

239 was the second most-abundant lineage among active organisms, indicating that anoxygenic

240 photosynthesis was still possible at this sediment depth (and/or bioturbation contributed to in-

241 mixing from more photosynthetically active surface layers).

242 Within the 10-20 mm depth zone, a post-BONCAT embedded section from a depth of 12

$243 \mathrm{~mm}$ was examined by correlative microscopy (Figs. 3-4). Twenty-two mineral grains were

244 analyzed; as above, the vast majority of grains were quartz, and the microbes associated with non-

245 quartz grains (in particular, orthoclase) had a higher proportion of anabolically active constituents

246 (Table 2). Across all mineral types, exterior organisms were more spatially constrained to surfaces

247 than the analyzed section from $7.6 \mathrm{~mm}$ : $40.5 \%$ were located within $5 \mu \mathrm{m}$ of mineral grains

248 compared to $27.8 \%$ in the top layer. Some of the highest concentrations of active cells were

249 associated not with well-defined minerals, but rather with heterogeneous patches that include small

250 particles of quartz, sodium, and iron (Fig. 3). In comparison with larger mineral grain interfaces, 
251 these particle assemblages offer greater chemically diversity and more potentially reactive surface

252 area, factors that may facilitate interactions among microbes.

253 Lower horizons BONCAT-FACS

254 The horizons from $20-60 \mathrm{~mm}$ did not have any corresponding sections analyzed by

255 microscopy, but the FACS and 16S rRNA gene sequencing data illuminate important trends in

256 community composition and metabolic activity with sediment depth. Among the eight most

257 abundant orders recovered, the sulfate-reducing Desulfobacterales and Desulfarculales accounted

258 for relatively consistent proportions of the active and inactive subsets throughout the core (Fig. 5).

259 The prominence of these lineages is consistent with previous observations that sulfate reduction is

260 the main remineralization metabolism in salt marsh sediments, accounting for roughly $80 \%$ of all

261 respiration at Great Sippewissett Marsh (Howarth and Teal, 1979). The more abundant

262 Desulfobacterales were more prevalent among anabolically active than inactive organisms at all

263 horizons, while the Desulfarculales frequently exhibited the opposite relationship. The latter order

264 consisted of the Desulfatiglans genus, whose abundance in subseafloor environments has been

265 attributed to its metabolic versatility in the degradation of aromatics (Jochum et al., 2018). In our

266 context, this versatility has seemingly enabled the genus to persist throughout the core, but the cost

267 of a diverse metabolic portfolio could be substantial lag times in metabolic re-routing or extended

268 periods of quiescence for organisms whose metabolic substrate is not present at a given time.

269 Purple sulfur bacteria Chromatiales, as anticipated, comprised a decreasing proportion of

270 active cells down-core in the absence of light. However, it was the most abundant order in several

271 inactive fractions, suggesting that purple sulfur bacteria may be among the larger microbial

272 contributors of organic matter to deeper sediments. Cellvibrionales and Rhodobacterales were

273 found at higher relative abundance in active than inactive fractions at the top of the core, but the 
274 opposite was true below $20 \mathrm{~mm}$ depth. Cellvibrionales have traditionally been considered

275 oligotrophs, but some members of the order contain sulfur oxidation pathways and others can grow

276 photoheterotrophically (Spring et al., 2014, 2015); this diversity of environments may explain their

277 relatively consistent presence among both active and inactive sequences throughout the core.

278 Rhodobacterales are noted early colonizers of particles (Dang et al., 2008); one of the most

279 prominent genera detected throughout the core was Rubribacterium, a non-sulfur purple bacterium

280 that is a facultative aerobe (Boldareva et al., 2009). These traits help explain the order's presence

281 at all horizons and its decrease in the active fraction with depth.

282 The observed vertical profile of Pirellulales sequences is consistent with aerobic

283 chemoorganotrophs (Schlesner et al., 2004) which may have been deposited onto the sediment

284 surface, metabolically inactivated quickly upon burial and the onset of anoxic conditions, and

285 potentially scavenged by the anoxic heterotrophs. Sphingobacterales are typically associated with

286 carbon remineralization in oxic soils (Fierer et al., 2007), but they do retain fermentation-

287 associated genes (Hester et al., 2018) that may explain their presence among the active cell fraction

288 we recovered from below $10 \mathrm{~mm}$ depth.

289 Deepest horizon BONCAT-FACS \& Correlative Microscopy

290 The deepest section used for correlative microscopy analysis was at a depth of $60.7 \mathrm{~mm}$.

291 Prior to cell extraction and sorting, a diverse range of fermentative lineages was observed, 292 including Anaerolinaceae (6.4\% of bulk sequences), Clostridia (2.6\%), and Bacteroidia (13\%).

293 Few sequences from putative methanogens were observed, potentially due to primer bias (Bahram

294 et al., 2019), seasonality (Buckley et al., 2008), and the presence of abundant sulfate-reducing

295 bacteria and a range of homoacetogens that may be more successful at attaining hydrogen

296 (Oremland and Polcin, 1982; Ye et al., 2014). In this horizon, the majority of observed (87.9\%) 
297 and sorted (85.5\%) cells were anabolically inactive (Table 2). The active and inactive communities

298 exhibited similar richness, but the active community had higher evenness (Fig. S3) and included a

299 comparatively higher relative proportion of Desulfobacterales, Bacteroidia, Clostridia, and

300 Anaerolinaceae.

301 Forty-two mineral grains were observed in the fluorescence microscopy field of view,

302 which also contained the highest abundance of small mineral particles and heterogenous patches

303 of the three sections (Figs. 6-7), potentially due to the diagenetic processes that accompany burial

304 and longer residence times within the sediment column (Curtis, 1987). Despite the high abundance

305 of associated mineral interfaces across a range of spatial scales, this horizon exhibited the lowest

306 microbial abundance and the lowest proportion of anabolically active organisms. This observation

307 is consistent with commonly observed trends in sediments, where electron acceptor depletion and

308 the progressive loss of labile carbon with depth can lead to energetically constrained conditions

309 (Blume et al., 2002; Jörgensen et al., 2002; Stone et al., 2014).

310 In the $60.7 \mathrm{~mm}$ horizon, quartz grains had the lowest cell abundances per unit surface area

311 and volume of the three examined sections, while orthoclase and plagioclase had higher-than-

312 average biomass densities (Table 2). The proportion of anabolically active organisms, however,

313 was not substantially different among distinct mineral types, suggesting that cells adhere more

314 strongly to plagioclase and orthoclase grains, and/or that quartz is more readily degraded during

315 diagenesis, disrupting surficial microbial association. This horizon also exhibited the highest

316 proportion of cells located inside mineral grains $(37.8 \%)$, an observation that could reflect the

317 extensive remineralization of external biomass with burial (Mackin and Swider, 1989).

318 Compiling findings across horizons 
When integrating sequencing and microscopy data across all horizons, intriguing trends of anabolic activity, diversity, and spatial arrangement emerged. With increasing depth into the

321 sediment, where geochemical and thermal conditions were more stable, alpha diversity metrics of

322 bulk pre-extraction communities revealed a decrease in richness but increase in evenness (Fig. S3).

323 Among the anabolically active and inactive communities, no substantial change in the number of

324 distinct ASVs with depth was observed, but the evenness of their distribution increased down-core

325 for the active constituents. This pattern may reflect a wider range of available niches with fewer

326 dominant lineages below the photic zone, as organic matter is remineralized through a range of

327 metabolic routes, making these deeper communities' emergent effects more resistant to

328 environmental changes (Wittebolle et al., 2009).

Beta diversity analysis revealed a clear separation of active and inactive communities,

331 and that anabolic activity is not a random process (Fig. S4). Furthermore, for both active and 332 inactive communities, the closer two sediment horizons were in depth, the more similar their 333 community compositions. This trend likely reflects depth-based gradients that form the energetic

334 basis for metabolic activity, as well as the burial process in which a given horizon's community 335 represents the confluence of local selective pressures operating on an assemblage of organisms 336 "imported" from above or from below due to tidal pumping.

337 At each of the three horizons examined through correlative microscopy, quartz was the 338 dominant mineral type, yet microbial communities associated with quartz grains had the lowest 339 proportion of anabolically active members. Other mineral types - such as orthoclase, plagioclase, 340 and rutile - had a broader set of cations ( $\mathrm{Al}, \mathrm{Ti}, \mathrm{K})$ that may have offered additional electron 341 transfer or nutrient acquisition opportunities for active cells. With increasing sediment depth, 
342 organisms were more likely to be located inside mineral grains, and these "internal" cells were

343 increasingly likely to be anabolically active compared with their "external" counterparts (Table

344 2): at $7.6 \mathrm{~mm}, 12 \mathrm{~mm}$, and $60.7 \mathrm{~mm}$ depth, internal organisms were $2.1 \%, 24 \%$, and $45 \%$ more

345 likely to be active than those outside minerals. These observations are consistent with a more stable

346 intra-mineral environment that may be less susceptible to predation, particularly in the more

347 energetically constrained anoxic sediment horizons.

\section{Conclusions}

The biological community of LSSM sediment demonstrated notable differences in its

350 composition, anabolic activity patterns, spatial arrangements, and mineralogical associations at the

351 three distinct horizons analyzed in this study. Following incorporation of HPG into new biomass

352 during a 3.7-day in situ incubation experiment, correlative microscopy, BONCAT-FACS, and

353 sequencing demonstrated that the most prevalent active constituents shifted from sulfur cycling

354 phototrophic consortia in the surficial horizon, to sulfate-reducing bacteria likely oxidizing a range

355 of organic compounds, to a range of fermentative lineages in the lower horizons. We observed a

356 rapid decay in the proportion of active organisms from $\sim 60 \%$ in the top $\mathrm{cm}$ to between $10-25 \%$ in

357 the horizons between 2-7 cm depth, offering a quantifiable reflection of the shift to the dark, anoxic

358 environment. By embedding sediment cores in resin, we mapped biomass and mineral grains with

359 microscale resolution and found that, on average, organisms were more distant from mineral grain

360 surfaces in the uppermost horizon, and most likely to be found inside mineral grains in the

361 lowermost horizon. Plagioclase, orthoclase, and rutile minerals recruited more abundant

362 communities that contained a higher proportion of anabolically active organisms compared with

363 quartz grains. Taken together, these findings give the impression of a more spatially and 
364 metabolically expansive community in surface sediments, fueled by sunlight and a range of

365 available niches, that is streamlined by burial and mineralogical weathering.

366 This benchmark study presents a promising new approach for exploring the anabolic

367 activity of a complex microbial community by mapping the precise spatial configuration of

368 anabolically active organisms within mineralogically heterogeneous salt marsh sediment through

369 correlative fluorescence and electron microscopy, while simultaneously identifying active

370 organisms in neighboring sediment with BONCAT-FACS and 16S rRNA gene sequencing. The

371 structure, activity, and evolutionary trajectory of complex microbial communities are determined

372 by the interactions between biotic and abiotic components of an ecosystem. Spatial relationships

373 are a powerful indication of these interactions, particularly in concert with the identification of

374 metabolically active organisms. Looking forward, the incorporation of rRNA-targeted FISH into

375 this workflow would enable a more direct connection between microbe-mineral spatial

376 arrangements and taxonomically constrained activity patterns. Improved approaches for

377 understanding microscale ecosystems in a new light, such as those presented here, reveal

378 environmental parameters that promote or constrain metabolic activity and clarify the impact that

379 microbial communities have on our world.

380 Experimental Procedures

381 Incubation Chamber Construction

382 Customized chambers were constructed to enable in situ incubation with HPG-infused

383 fluid. Glycol-modified polyethylene terephthalate (PETG) tubes (1" outer diameter, 0.75 " inner

384 diameter, McMaster-Carr, Elmhurst, IL) were cut to $\sim 30 \mathrm{~cm}$ length. PETG was used because of

385 its low gas permeability and high optical transparency (Thermo Fisher Scientific), properties that

386 diminished oxygen penetration of subsurface sediments during recovery and transport while 
retaining light availability for surface-exposed organisms during the incubation period. The lower opening of each tube was beveled with sandpaper (giving the tube a sharp interior edge) to minimize the effects of compaction on collected material when pressing the tube into the sediment. the incubation tube; by removing them, percolation was enabled.

At certain times during the incubation, gas-permeable, liquid-impermeable conditions were required. This was achieved with 0.01 "'-thick silicone polydimethylsiloxane (PDMS) membranes (Interstate Specialty Products, Sutton, MA) that were secured between the end of the tube and the screw-top lid. Holes were poked into the Falcon tube lid with a needle to facilitate gas exchange at both the top and bottom of the incubation volume. All materials were thoroughly cleaned with $70 \%$ ethanol and 60 minutes of UV light exposure prior to use.

Sample Recovery, HPG Addition, and In Situ Incubation Sippewissett Salt Marsh (LSSM) in Falmouth, MA. The "Berry Pond" at $41.5758^{\circ}$ latitude, -

$40370.6394^{\circ}$ longitude (Fig. S1) was selected for sampling due to its extensive heritage in 404 environmental microbiology research, which would provide greater context to our studies. The 405 work presented here pertains to sediment cores collected with five customized incubation tubes 406 (see Table S1). Cores BM and BS were treated with HPG for BONCAT analysis; CM and CS were 407 control samples with no HPG exposure. BM and CM were used for microscopy analysis; BS and 408 CS were used for community analysis by FACS and subsequent 16S rRNA gene sequencing of 409 anabolically active and inactive populations. An additional control of homogenized 0-10 $\mathrm{cm}$ depth 
410 LSSM sediment (core AM) was autoclave-sterilized and then incubated with HPG solution 411 (described below) for 89 hours in the lab.

412 At approximately 07:00 (24-hour clock), the tubes were pressed into the sediment, 413 collecting $\sim 10-12 \mathrm{~cm}$ of sediment; upon removal, an autoclave-sterilized plug of glass wool (Fisher 414 Scientific) was inserted into the bottom of the tube. (See Fig. 8 for a schematic of the collection 415 and sample processing approach.) Permeable and intact Falcon tube caps were twisted onto the top 416 and bottom of the chamber, respectively. At approximately 7:30, the incubation chambers were 417 placed in an anoxic glove box $\left(3.5 \% \mathrm{H}_{2}, 20 \% \mathrm{CO}_{2}, 76.5 \% \mathrm{~N}_{2}\right)$, the caps were removed, and fluid 418 replacement began. Based on the permeability of the sediment and the glass wool plug, fluid 419 moved through the sediment column at a rate of $\sim 0.3 \mathrm{~mL}$ per minute per $\mathrm{cm}^{2}$.

420 Next, the sediment water was replaced with fluid containing HPG (Click Chemistry Tools, 421 Scottsdale, AZ) for anabolic uptake. $50 \mu \mathrm{M}$ HPG was dissolved in $0.22 \mu \mathrm{m}$-filtered Berry Pond 422 water and stored in an anoxic chamber overnight. (The appropriate concentration of HPG and dye 423 for subsequent visualization was determined from a previous study (Hatzenpichler and Orphan, 4242015 ) and a series of tests using 5-500 $\mu \mathrm{M}$ HPG and 0.5-50 $\mu \mathrm{M}$ dye; see Fig. S5.) Four to six 425 column volumes of HPG solution were added dropwise to the top of the column at roughly the 426 same rate as fluid leaked out the bottom, resulting in a full replacement of permeable volume with 427 HPG solution in a manner that did not substantially change the overlying pressure experienced by 428 the sediment. PDMS membranes were secured to the top and bottom of the incubation chamber 429 with the same Falcon tube caps as before, and the samples were transported back to the Berry Pool. $430 \quad$ At the precise location of sample collection, the incubation chambers were placed back 431 into the marsh sediment at 12:00. Immediately prior to deposition, holes were poked in the bottom 432 caps (but not the membrane) to ensure the full length of the sediment was in gaseous equilibrium 
433 with its surroundings, but that the HPG solution remained contained. The tubes were aligned such

434 that the water-sediment interface matched that of the sediment in the Berry Pool. The samples were

435 left to incubate in the marsh for 89 hours (retrieval time September $30^{\text {th }}, 05: 00$ ). The incubation

436 timing was determined by experiments of LSSM sediments demonstrating apparent saturation of

437 BONCAT-positive signal after 88 hours (Fig. S6).

438 Throughout the process described above, the incubation chambers were checked for leaks.

439 Overlying water levels were marked on the tube exterior after sample recovery, after HPG fluid

440 introduction, and upon re-introduction to the marsh; experiments only proceeded if no change in

441 water level was observed.

$442 \quad$ Preparation of Cores for Microscopy

443 Our core preparation expanded on a protocol that was first used in the analysis of volcanic

444 fumarole soils (Marlow et al., 2020). Cores BM and CM were chemically fixed, dehydrated,

445 embedded in resin, sectioned, and stained for analysis by fluorescence and electron microscopy.

446 Four to six column volumes of 3\% PFA solution (in $0.22 \mu \mathrm{m}$-filtered Berry Pond water) were

447 percolated through the samples to fully replace the HPG solution. An intact bottom cap was

448 secured to the bottom of the core tube, and samples were incubated at room temperature for four

449 hours. A dehydration series consisting of $4-6$ column volumes each of $50 \%, 80 \%$, and $96 \%$ ethanol

450 (in $0.22 \mu \mathrm{m}$-filtered Berry Pond water) was performed.

451 Following dehydration, sediment samples were embedded in LR White resin (hard grade,

452 Electron Microscopy Sciences, Hatfield, PA), which was selected for its low viscosity and minimal

453 background signal under the wavelengths used for fluorescence microscopy. LR White has been

454 used in a number of similar applications, including correlative microscopy of animal tissue

455 (Hegermann et al., 2019), plant tissues (Bell et al., 2013), low diversity microbial biofilms 
456 (Knierim et al., 2012), carbonate microbialites (Gérard et al., 2013), and marine sediment

457 (McGlynn et al., 2018). Four to six column volumes of 100\% liquid resin were percolated through

458 the dehydrated columns, and intact Falcon tube caps were secured on the bottom to avoid leakage.

459 The samples were then placed in an incubation oven at $60{ }^{\circ} \mathrm{C}$ for 36 hours to cure.

The solidified sediment columns were sectioned with a diamond saw (Model 650, South

461 Bay Technology, San Clemente, CA) and an ethanol-sterilized PELCO diamond wafering blade

462 (\#812-332, Ted Pella, Inc., Redding, CA) spun through ultrapure Milli-Q cooling water. Sections

463 were submerged in a $5 \mu \mathrm{M}$ Cy3 Picolyl Azide dye (Click Chemistry Tools, Scottsdale, AZ) click

464 staining solution (Hatzenpichler and Orphan, 2015) in an anoxic chamber for 60 minutes in the

465 dark. Afterwards, they were removed from the chamber, washed three times with sterile PBS

466 solution, incubated in 5x SYBR Green I (referred to hereafter as "SYBR green"; Life

467 Technologies, ThermoFisher, Waltham, MA) in the dark at room temperature for 15 minutes,

468 rinsed three times with sterile PBS, and left to air dry in the dark prior to imaging. All downstream

469 correlative microscopy was performed on areas as far from the outer edge of the sediment core as

470 possible $(\sim 8 \mathrm{~mm})$ in order to minimize the effects of the coring process on the analyzed area.

$471 \quad$ Fluorescence Microscopy

472 Fluorescence imaging of sectioned samples was done with a LSM 880 confocal laser

473 scanning microscope (Zeiss, Oberkochen, Germany) equipped with a gallium arsenide phosphide

474 (GaAsP) detector, a 20x objective lens, and DI water immersion. Argon and DPSS lasers provided

475 excitation at 458, 488, 514, and $561 \mathrm{~nm}$ wavelengths. Detected emission windows were 510-561

$476 \mathrm{~nm}$ for SYBR green and 564-669 $\mathrm{nm}$ for Cy5. Reflected light from the $488 \mathrm{~nm}$ laser was also

477 captured to link sample features between confocal and electron microscopy images as reference

478 points. Imaging was done with the Zen 2.3 SP1 program (Zeiss, Oberkochen, Germany). Focus 
was adjusted manually for each field of view. 1,024 x 1,024 pixel frames were acquired with a pixel dwell time of $32.77 \mu \mathrm{sec}$ and a pixel size of $240 \mathrm{~nm}$. Four line-based scans were averaged to

481 generate each image. Gain settings were set to minimize background and nonspecific signals. For

482 the SYBR channel, 800 master gain and 1.3 digital gain were used; these parameters were 680 and

483 1.2, respectively, for the Cy3 channel.

484 Electron Microscopy \& Energy Dispersive X-Ray Spectroscopy

After fluorescence microscopy, each section was dipped in a dehydration series of $50 \%$,

$80 \%$, and $100 \%$ ethanol solutions (balance Milli-Q water). The sample was then mounted on a

SEM sample holder with double-sided carbon tape and sputter-coated with $5 \mathrm{~nm}$ of Pt/Pd (EMS

SmartSEM software, secondary electron images were collected at a voltage of $12 \mathrm{kV}$ using an

490 Everhart-Thornley detector. The voltage was increased to $20 \mathrm{kV}$ for elemental analysis. A silicon

491 drift detector was used, and data were processed with the EDAX Genesis software (Ametek,

492 Berwyn, PA). Elemental maps were used for visualization purposes, supported by quantitative

493 area-based scans to inform mineral identification by X-Ray diffraction.

494 X-Ray Diffraction

X-ray diffraction (XRD) was performed using an X'Pert3 powder diffractometer by

496 Panalytical using a $\mathrm{Cu} \mathrm{K} \alpha$ source to scan from 5-70 $2 \theta$. The sample was prepared as a packed 497 powder and was scanned wobbling the sample stage at $0,-1$, and +1 degrees. The final scan was 498 an average of these three scans. Phase identification and semi-quantitative analysis of 499 diffractograms was performed using the HighScore Plus software by Panalytical (Malvern 500 Panalytical, Malvern, United Kingdom).

$501 \quad$ Image Processing 
503 (Schindelin et al., 2012). We used the DeconvolutionLab2 plugin with a point spread function

504 calculated by the Zen program (based on our specific imaging parameters) and five iterations of

505 the Richardson-Lucy algorithm (Richardson, 1972; Lucy, 1974). The Despeckle program was used

506 for denoising.

To link the location of fluorescent signal with the high-resolution textural information enabled by electron microscopy, image co-registration was performed using the bUnwarpJ

509 algorithm using the following parameters: accurate registration, very coarse initial deformation, 510 super fine final deformation, 0.1 divergence weight, 0.1 curl weight, 3.5 landmark weight, 0.0

511 image weight, 10 consistency weight, and 0.1 stop threshold. The SEM/EDS images were 512 designated as the "target" images onto which fluorescence images were mapped, and 513 approximately one landmark per $10,000 \mu \mathrm{m}^{2}$ was designated on the SEM and reflected light

514 fluorescence images. The other two fluorescence channels (SYBR green and Cy3) were anchored 515 to the reflected light layer to accurately co-register SYBR and BONCAT signals with SEM and 516 EDS data (see Fig. S7).

517 Cell counting and distance relationships were analyzed in Matlab R2018b. Red (Cy3, 518 BONCAT) and green (SYBR green, all cells) channels were separated and converted to binary 519 images at a manually determined global threshold of 0.04. Single pixels were eliminated to remove 520 noise, and individual regions of interest were designated by applying a watershed transform with 521 four-degree connectivity (e.g., pixels that only touched at corners rather than edges were not 522 counted as the same object). Finally, centroid holes were filled, and remaining shapes were counted 523 as "organisms". An organism was recorded as anabolically active if at least one of its pixels 524 fluoresced both red and green after being subjected to the image analysis pipeline. 
Organism concentrations were calculated by counting the number of organisms in the relevant field of view and dividing that number by the volume sampled through fluorescence

527 microscopy. This volume was determined using the $\mathrm{x}$ and $\mathrm{y}$ dimensions of the microscopy footprint 528 and the $\mathrm{z}$ dimension $(1.66 \mu \mathrm{m})$ empirically established by an average of the SYBR green and Cy3 529 signal transmission distances through the resin, as shown in Fig. S8.

530 Distances between organisms and mineral surfaces were measured by first manually 531 tracing the outlines of mineral grains in Adobe Photoshop using the high-resolution SEM images 532 and converting the resulting image into a binary image in Matlab. Next, we calculated the shortest 533 Euclidean distance from each organism's outer surface to the perimeter of each mineral. The 534 shortest distance and identity of the associated mineral were recorded. Because this analysis was 535 restricted to a two-dimensional cross-sectional view of three-dimensional mineral grains, distances 536 from mineral grain-internal cells to the mineral surface are not reported. To exclude cells that had 537 been dislodged during the embedding and sectioning process, all cells more than $70 \mu \mathrm{m}$ away from 538 the nearest mineral surface were omitted from distance-based calculations. This cutoff, which 539 removed $6.2 \%$ of all organisms from spatial analysis only, was based on a distance histogram to 540 determine outliers (Fig. S9) and is within the range of biofilm thicknesses associated with silica 541 mineral surfaces (which represented the majority of observed mineral grains) (Ye et al., 2015).

542 During sectioning, a few mineral grains were plucked from the resin: three in the top section, two 543 in the middle, and three in the bottom. Organisms associated with these grains that remained in the 544 resin were not included in spatial analyses, and the overall loss of material indicates that cell 545 abundance values represent a lower bound. When normalizing organism abundances by mineral 546 surface area and volume, perimeters calculated from mineral grain outlines were used as a proxy 547 for surface area, and cross-sectional area was used as a proxy for grain volume. 
Fluorescence-Activated Cell Sorting (FACS)

Samples BS and CS were used for FACS and high-throughput 16S rRNA gene sequencing

to identify the subset of microorganisms that was anabolically active during the incubation period.

551 These sediment cores were shipped on ice from Massachusetts to Montana State University for

552 analysis (shipment took $\sim 19$ hours). Prior to shipment, the overlying liquid was removed and the

553 top $1.0 \mathrm{~cm}(+/-0.3 \mathrm{~cm})$ flocculent layer of sediment was transferred to a separate sterile tube to

554 avoid compression and inaccurate identification of horizons. Sterile glass wool was added to the

555 top of the incubation chamber to maintain core coherence during transport.

Upon arrival, each sediment core was carefully excised from the core sleeve using a

557 custom-built, sterilized plunger. Each core was divided into $1 \mathrm{~cm}$ increments, which were weighed,

558 transferred into Falcon tubes containing $10 \mathrm{~mL}$ of sterilized $1 \mathrm{xPBS}$, and stored at $4^{\circ} \mathrm{C}$ until cells

559 were extracted. Cells were extracted from each sediment layer using methods adapted from

560 Couradeau et al. (Couradeau et al., 2019) with the following modifications. For each sediment

561 layer, $1 \mathrm{~mL}$ of the slurry was diluted with $5 \mathrm{~mL}$ of sterile PBS in a $15 \mathrm{~mL}$ Falcon tube with

562 Tween 20 (final concentration $0.02 \%$ ). The cell extraction slurry was placed on a benchtop vortexer

563 at maximum speed for 5 minutes. Large sediment particles were pelleted via centrifugation at 500

$564 \mathrm{x}$ g for 55 minutes. Cells from $700 \mu \mathrm{L}$ of the supernatant were pelleted in a $1.5 \mathrm{~mL}$ microcentrifuge

565 tube by centrifugation at $16,000 \mathrm{x}$ g for 5 minutes. The supernatant was carefully removed by

566 pipette before the click reaction was performed directly on the cell pellet. Extraction blanks were

567 performed without any added sediment in parallel with cell extractions to test for reagent

568 contamination.

569 The click reaction solution was prepared in a large volume in order to stain all samples

570 using the same solution. The reaction solution was prepared as previously described 
571 (Hatzenpichler and Orphan, 2015) with the addition of a general DNA stain, SYBR green

572 (ThermoFisher Scientific, Invitrogen, Eugene, OR, USA) to counterstain all cells. The solution

573 contained $5 \mathrm{mM}$ aminoguanidine hydrochloride (Sigma Aldrich), $5 \mathrm{mM}$ sodium $L$-ascorbate

574 (Sigma Aldrich), $100 \mu \mathrm{M}$ copper sulfate pentahydrate (Sigma Aldrich), $500 \mu \mathrm{M}$ THPTA (Click

575 Chemistry Tools), $12 \mu \mathrm{M}$ Cy5 picolyl-azide dye (Click Chemistry Tools, Scottsdale, AZ), and 1x

576 SYBR green in PBS. The solution was vortexed, and $200 \mu \mathrm{L}$ was transferred to each cell pellet

577 and mixed with cells by pipetting up and down. The click reaction solution and cells were

578 incubated for $30 \mathrm{~min}$ in the dark on a slow rotator at room temperature. Click reaction solution

579 was washed from cells by three cycles of (1) centrifugation at 17,000 x g for 5 minutes, (2) removal

580 of supernatant by pipette, and (3) resuspension in sterile PBS. The final cell pellet was resuspended

581 in $700 \mu \mathrm{L}$ of sterile $1 \mathrm{x}$ PBS. Prior to cell sorting, the cell suspension was passed through a $35 \mu \mathrm{m}$

582 filter cap (BD-falcon $5 \mathrm{~mL}$ tube with cell strainer cap, CorningTM, Corning, NY, USA) to remove

583 any remaining large debris.

A Sony SH800S FACS (Sony Biotechnology, San Jose, CA) was used to sort cells via a

$58570 \mu \mathrm{m}$ chip. The cell sorter was set to detect the SYBR green dye on the green channel (excitation

$586488 \mathrm{~nm}$ ) and Cy5 dye on the red channel (excitation $638 \mathrm{~nm}$ ). The first two gates were drawn on

587 forward scatter and back scatter properties to remove any large particles or noise. The third gate

588 was drawn on SYBR green positive ("sortable cells") events, with the assumption that this gate

589 captured all cells. Only SYBR+ cells were gated to be either BONCAT positive ("active cells") or

590 BONCAT negative ("inactive cells") on the Cy5 channel. Core CS, the no-HPG control, was used

591 to determine the cutoff between BONCAT positive and negative fractions. Gates were drawn

592 conservatively to minimize the possibility for false positives or false negatives in any gate (Fig.

593 S2). For each of three biological replicates for each cell fraction ("active cells" and "inactive 
594 cells"), $1 \times 10^{6}$ cells were sorted into $1.5 \mathrm{~mL}$ microcentrifuge tubes containing $500 \mu \mathrm{L}$ of sterile $1 \mathrm{x}$

595 PBS. Sorted cells were stored at $4{ }^{\circ} \mathrm{C}$ for up to six hours before being centrifuged at $17,000 \mathrm{xg}$ for

5965 minutes to pellet and then resuspended in $20 \mu \mathrm{L}$ of nuclease free water and frozen at $-80^{\circ} \mathrm{C}$ until

597 DNA extraction and downstream processing.

$598 \quad$ 16S rRNA Gene Amplicon Sequencing

599 To capture the bulk microbial community from each sediment layer in core BS, DNA was

600 extracted from $500 \mu \mathrm{L}$ sediment/1x PBS slurry from each layer using the FastDNA Spin Kit for

601 Soils (MP Biomedicals, Irvine, CA) following the manufacturer's instructions. A blank DNA

602 extraction was processed in parallel with bulk sediment extractions to check for contaminants.

603 DNA was extracted from sorted cells and processing blanks as previously described (Reichart et

$604 a l ., 2020)$. Briefly, cell suspensions were transferred to a 96-well microtiter plate and sealed with

605 sterile adhesive foil before being subjected to three freeze-thaw cycles $\left(-80{ }^{\circ} \mathrm{C}\right.$ for $20 \mathrm{~min}, 99^{\circ} \mathrm{C}$

606 for $10 \mathrm{~min}$ ), with brief centrifugation prior to each freezing step. 16S rRNA genes of bacteria and

607 archaea were amplified following the Earth Microbiome protocol (Thompson et al., 2017) using

608 revised primers 515F (Parada et al., 2016) and 806R (Apprill et al., 2015) added directly into the

609 extracted DNA microtiter plates. These primers were designed prior to the discovery of several

610 new lineages related to the DPANN and Asgard archaea superphyla (Baker et al., 2020) and thus

611 do not capture the entire archaeal diversity (Bahram et al., 2019; Pausan et al., 2019). However,

612 their extensive use in environmental microbiology laboratories around the world enables

613 comparability between studies (Gilbert et al., 2014; Thompson et al., 2017) and within our own

614 laboratories. The final PCR volume was $37.5 \mu \mathrm{L}$ and consisted of $15 \mu \mathrm{L}$ Invitrogen Platinum Taq

615 II $2 X$ Master Mix, $0.75 \mu \mathrm{L} 515 \mathrm{~F}$ primer $(10 \mu \mathrm{M}$; final: $0.2 \mu \mathrm{M}), 0.75 \mu \mathrm{L}$ 806R primer $(10 \mu \mathrm{M}$;

616 final: $0.2 \mu \mathrm{M}$ ), and $1 \mu \mathrm{L}$ nuclease-free water added directly into the microtiter plates containing 
617 the $20 \mu \mathrm{L}$ of lysate. The thermocycler conditions were: $94{ }^{\circ} \mathrm{C}$ for 3 minutes followed by 28 cycles

618 of $94{ }^{\circ} \mathrm{C}$ for $45 \mathrm{sec}, 50{ }^{\circ} \mathrm{C}$ for $60 \mathrm{sec}$, and $72{ }^{\circ} \mathrm{C}$ for $90 \mathrm{sec}$ before a final elongation step at $72{ }^{\circ} \mathrm{C}$

619 for 10 minutes. A negative PCR control was processed in parallel using nuclease-free water instead

620 of extracted DNA to check for PCR contaminants. PCR products were purified using AMPure XR

621 beads (Beckman Coulter) following the manufacturer's protocol with a final elution in $40 \mu \mathrm{L}$

622 nuclease free water.

623 Afterwards, a second PCR to attach barcodes and sequencing adapters to the 16S rRNA

624 gene amplicons was performed. The final volume of the PCR was $25 \mu \mathrm{L}$ and contained $5 \mu \mathrm{L}$

625 purified, amplified DNA, $12 \mu \mathrm{L}$ Invitrogen Platinum Taq II 2X Master Mix, $2.5 \mu \mathrm{L}$ i5 primer

626 (final: $0.25 \mu \mathrm{M}$ ), $2.5 \mu \mathrm{L}$ i7 primer (final: $0.25 \mu \mathrm{M}$ ), and $2.5 \mu \mathrm{L}$ water. The thermocycler

627 conditions were $95^{\circ} \mathrm{C}$ for 3 minutes followed by 8 cycles of $95^{\circ} \mathrm{C}$ for $30 \mathrm{sec}, 55^{\circ} \mathrm{C}$ for $30 \mathrm{sec}$,

628 and $72{ }^{\circ} \mathrm{C}$ for $30 \mathrm{sec}$, followed by a final elongation step at $72{ }^{\circ} \mathrm{C}$ for 5 minutes. A PCR product

629 purification was performed as described above using AMPure XR beads. Finally, purified PCR

630 products were checked for appropriate length by gel electrophoresis in 1\% agarose. Amplicons

631 were quantified in triplicate using Quant-iT Picogreen dsDNA Assay (Invitrogen) following the

632 manufacturer's protocol and measured on a Biotek Synergy H1 Hybrid microplate reader.

633 Samples were pooled at $10 \mathrm{ng}$ DNA each, and the final pooled sample was purified and

634 concentrated with the QIAquick PCR purification spin column kit (Qiagen) following the

635 manufacturer's guidelines. Sequencing was performed by Laragen Inc. (Culver City, CA) using

636 Illumina 2x250 paired end read MiSeq sequencing. Sequences have been archived at NCBI

637 Genbank under the Bioproject ID PRJNA643437.

638 Sequence analysis 
Primers were removed from demultiplexed sequences using cutadapt (Martin, 2011) with

640 a max mismatch of $2 \mathrm{bp}$ and requirement that primers must be present on both forward and reverse

641 reads to maintain the read pair in the dataset. Unpaired primer-free reads were processed in $D A D A 2$

642 (Callahan et al., 2016) run in the R environment. Reads were first trimmed to 220 bp for forward

643 reads and $170 \mathrm{bp}$ for reverse reads, then were filtered with default settings of $\operatorname{maxN}=0$,

$644 \operatorname{maxEE}=\mathrm{c}(2,2)$, trunc=2, and rm.phix=TRUE. Denoising of reads was conducted with $D A D A 2$ 's

645 error model calculated on randomly sampled reads from the entire dataset. Forward and reverse

646 reads were merged with a $20 \mathrm{bp}$ minimum overlap and no mismatches. Chimeras were removed

647 within $D A D A 2$ using the "consensus" method. Taxonomy of the amplicon sequence variants

648 (ASVs) was assigned using the SILVA132 database (Quast et al., 2012). To remove contaminating

649 sequences, R package decontam (Davis et al., 2018) was implemented using the "prevalence"

650 model with a threshold of 0.7 , which removed 150 of the total 11,014,619 ASVs. Five samples

651 with fewer than 10,000 sequences were removed from the dataset. Further normalization of read

652 count per sample was performed using the R package metagenomeSeq (Paulson et al., 2013),

653 which builds a statistical model to account for undersampling. Diversity metrics including

654 Shannon's diversity index, Bray-Curtis similarity, and non-metric multidimensional scaling

655 (NMDS) were calculated in the Phyloseq (McMurdie and Holmes, 2013) R package.

656 Quality Control

Analysis of samples subjected to the full experimental treatment alongside control samples

658 allowed us to validate our procedures. Comparing sample BM with AM indicated that neither

659 SYBR nor Cy3 signals were attributable to background fluorescence or non-specific binding of

660 HPG or the dyes; across five fields of view from both samples, 96.6\% of SYBR-active objects and

$66197.1 \%$ of Cy3-active objects were present in sample BM. Comparing sample BM with CM 
revealed that HPG did not affect SYBR signal but was required for BONCAT signal: $44.8 \%$ of

663 SYBR-active objects and $97.2 \%$ of Cy3-active objects were found in sample BM. the empirical biases that may result was a key priority. Daily fluctuations of the Berry Pool water level, which ranges from $\sim 5-30 \mathrm{~cm}$ water depth over the course of a tidal cycle, consistently

667 introduce and remove transient organisms that may not be physically associated with sediment particles. Nonetheless, it is possible that the percolation of fluids through the incubation chambers might transport microbial cells outside of their naturally-occurring habitats. To test this possibility, we introduced $1 \mathrm{~mL}$ of $1 \times 10^{9} / \mathrm{mL} 1 \mu \mathrm{m}$ diameter YG carboxylate fluorescent microspheres

671 (Polysciences, Warrington, PA) to the overlying water of a LSSM sediment core. These 672 microspheres are commonly used to simulate microorganism transport and constrain 673 contamination in sediments, soils, and subsurface environments (House et al.; Smith et al., 2000;

674 Goeppert and Goldscheider, 2011; Bang-Andreasen et al., 2017; Labonté et al., 2017; Daly et al., 2018). Following microsphere addition, the core was treated identically to the BM sample. Flowthrough liquid fractions were collected and deposited on $0.22 \mu \mathrm{m}$ polycarbonate filters, and

677 multiple horizons were sectioned and examined with fluorescence microscopy. Bead counts over

678 five representative fields of view at $10.7 \mathrm{~mm}$ above the sediment-water interface, $2.0 \mathrm{~mm}$ depth,

$6795.3 \mathrm{~mm}$ depth, $9.8 \mathrm{~mm}$ depth, and $23.3 \mathrm{~mm}$ depth (Fig. S10), as well as 16 liquid fractions, were 680 averaged and scaled by the overall cross-sectional area of the core. Z-axis transmission of bead 681 fluorescence under confocal microscopy examination was $8.75 \mu \mathrm{m}$. Linear interpolation of data 682 points indicated that $99.3 \%$ of beads remained above the $7.6 \mathrm{~mm}$ horizon, which was the shallowest 683 horizon used for microscopy analysis. Assuming a cell density of $10^{6} / \mathrm{mL}$ in the overlying water 684 and $30 \mathrm{~mL}$ of overlying water in the initial core sample, we calculate that $6 \times 10^{-3} \%$ and $8 \times 10^{-4} \%$ 
685 of cells detected in the $7.6 \mathrm{~mm}$ and $12 \mathrm{~mm}$ horizons, respectively, are attributable to entrained surface water cells. Because a horizon lower than $60.7 \mathrm{~mm}$ was not examined with the bead test,

687 an analogous figure is not attainable for the $60.7 \mathrm{~mm}$ horizon. However, given the trends observed

688 here, we believe the contribution from surface-entrained organisms is negligible. This analysis

689 gave us confidence in interpreting mineral-associated organisms as native to the observed sediment

690 horizons.

\section{Acknowledgements} declare no conflict of interest.

\section{References} et al. (2018) Environmental controls, emergent scaling, and predictions of greenhouse gas (GHG) fluxes in coastal salt marshes. Journal of Geophysical Research: Biogeosciences 123: 2234-2256.

Andersen, S.B., Marvig, R.L., Molin, S., Krogh Johansen, H., and Griffin, A.S. (2015) Longterm social dynamics drive loss of function in pathogenic bacteria. Proc Natl Acad Sci USA 112: 10756.

Angell, J.H., Peng, X., Ji, Q., Craick, I., Jayakumar, A., Kearns, P.J., et al. (2018) Community composition of nitrous oxide-related genes in salt marsh sediments exposed to nitrogen enrichment. Frontiers in microbiology 9: 170.

Apprill, A., McNally, S., Parsons, R., and Weber, L. (2015) Minor revision to V4 region SSU rRNA 806R gene primer greatly increases detection of SAR11 bacterioplankton. Aquatic Microbial Ecology 75: 129-137. 
Armitage, D.W., Gallagher, K.L., Youngblut, N.D., Buckley, D.H., and Zinder, S.H. (2012) Millimeter-scale patterns of phylogenetic and trait diversity in a salt marsh microbial mat. Frontiers in Microbiology 3: 293.

Bagert, J.D., Xie, Y.J., Sweredoski, M.J., Qi, Y., Hess, S., Schuman, E.M., and Tirrell, D.A. (2014) Quantitative, time-resolved proteomic analysis by combining bioorthogonal noncanonical amino acid tagging and pulsed stable isotope labeling by amino acids in cell culture. Molecular \& Cellular Proteomics 13: 1352-1358.

Bahram, M., Anslan, S., Hildebrand, F., Bork, P., and Tedersoo, L. (2019) Newly designed 16S rRNA metabarcoding primers amplify diverse and novel archaeal taxa from the environment. Environmental microbiology reports 11: 487-494.

Baker, B.J., De Anda, V., Seitz, K.W., Dombrowski, N., Santoro, A.E., and Lloyd, K.G. (2020) Diversity, ecology and evolution of Archaea. Nature Microbiology 1-14.

Bang-Andreasen, T., Schostag, M., Priemé, A., Elberling, B., and Jacobsen, C.S. (2017) Potential microbial contamination during sampling of permafrost soil assessed by tracers. Scientific reports 7: 1-11.

Bell, K., Mitchell, S., Paultre, D., Posch, M., and Oparka, K. (2013) Correlative imaging of fluorescent proteins in resin-embedded plant materiall. Plant physiology 161: 15951603.

Blume, E., Bischoff, M., Reichert, J., Moorman, T., Konopka, A., and Turco, R. (2002) Surface and subsurface microbial biomass, community structure and metabolic activity as a function of soil depth and season. Applied Soil Ecology 20: 171-181.

Boetius, A., Ravenschlag, K., Schubert, C.J., Rickert, D., Widdel, F., Gieseke, A., et al. (2000) A marine microbial consortium apparently mediating anaerobic oxidation of methane. Nature 407: 623.

Boldareva, E., Moskalenko, A., Makhneva, Z., Tourova, T., Kolganova, T., and Gorlenko, V. (2009) Rubribacterium polymorphum gen. nov., sp. nov., a novel alkaliphilic nonsulfur purple bacterium from an Eastern Siberian soda lake. Microbiology 78: 732-740.

Bowen, J.L., Byrnes, J.E., Weisman, D., and Colaneri, C. (2013) Functional gene pyrosequencing and network analysis: an approach to examine the response of denitrifying bacteria to increased nitrogen supply in salt marsh sediments. Frontiers in microbiology 4: 342.

Bowen, J.L., Morrison, H.G., Hobbie, J.E., and Sogin, M.L. (2012) Salt marsh sediment diversity: a test of the variability of the rare biosphere among environmental replicates. The ISME journal 6: 2014-2023.

Buckley, D.H., Baumgartner, L.K., and Visscher, P.T. (2008) Vertical distribution of methane metabolism in microbial mats of the Great Sippewissett Salt Marsh. Environmental Microbiology 10: 967-977.

Callahan, B.J., McMurdie, P.J., Rosen, M.J., Han, A.W., Johnson, A.J.A., and Holmes, S.P. (2016) DADA2: high-resolution sample inference from Illumina amplicon data. Nature methods 13: 581.

Cordero, O.X. and Datta, M.S. (2016) Microbial interactions and community assembly at microscales. Current opinion in microbiology 31: 227-234.

Cordero, O.X., Ventouras, L.-A., DeLong, E.F., and Polz, M.F. (2012) Public good dynamics drive evolution of iron acquisition strategies in natural bacterioplankton populations. Proceedings of the National Academy of Sciences 109: 20059-20064. 
Couradeau, E., Sasse, J., Goudeau, D., Nath, N., Hazen, T.C., Bowen, B.P., et al. (2019) Probing the active fraction of soil microbiomes using BONCAT-FACS. Nature Communications 10: 2770 .

Coyte, K.Z., Schluter, J., and Foster, K.R. (2015) The ecology of the microbiome: Networks, competition, and stability. Science 350: 663.

Curtis, C. (1987) Mineralogical consequences of organic matter degradation in sediments: inorganic/organic diagenesis. In Marine Clastic Sedimentology. Springer, pp. 108-123.

Daly, R.A., Wrighton, K.C., and Wilkins, M.J. (2018) Characterizing the Deep Terrestrial Subsurface Microbiome. In Microbiome Analysis. Springer, pp. 1-15.

Dang, H., Li, T., Chen, M., and Huang, G. (2008) Cross-ocean distribution of Rhodobacterales bacteria as primary surface colonizers in temperate coastal marine waters. Appl Environ Microbiol 74: 52-60.

Davis, N.M., Proctor, D.M., Holmes, S.P., Relman, D.A., and Callahan, B.J. (2018) Simple statistical identification and removal of contaminant sequences in marker-gene and metagenomics data. Microbiome 6: 226.

Dieterich, D.C., Link, A.J., Graumann, J., Tirrell, D.A., and Schuman, E.M. (2006) Selective identification of newly synthesized proteins in mammalian cells using bioorthogonal noncanonical amino acid tagging (BONCAT). Proceedings of the National Academy of Sciences 103: 9482-9487.

Dong, H., Jaisi, D.P., Kim, J., and Zhang, G. (2009) Microbe-clay mineral interactions. American Mineralogist 94: 1505-1519.

Fierer, N., Bradford, M.A., and Jackson, R.B. (2007) Toward an ecological classification of soil bacteria. Ecology 88: 1354-1364.

Gérard, E., Ménez, B., Couradeau, E., Moreira, D., Benzerara, K., Tavera, R., and López-García, P. (2013) Specific carbonate-microbe interactions in the modern microbialites of Lake Alchichica (Mexico). The ISME journal 7: 1997-2009.

Gilbert, J.A., Jansson, J.K., and Knight, R. (2014) The Earth Microbiome project: successes and aspirations. BMC biology 12: 69.

Goeppert, N. and Goldscheider, N. (2011) Transport and variability of fecal bacteria in carbonate conglomerate aquifers. Groundwater 49: 77-84.

Gómez-Pereira, P.R., Schüler, M., Fuchs, B.M., Bennke, C., Teeling, H., Waldmann, J., et al. (2012) Genomic content of uncultured Bacteroidetes from contrasting oceanic provinces in the North Atlantic Ocean. Environmental Microbiology 14: 52-66.

Gong, B., Wu, P., Ruan, B., Zhang, Y., Lai, X., Yu, L., et al. (2018) Differential regulation of phenanthrene biodegradation process by kaolinite and quartz and the underlying mechanism. Journal of hazardous materials 349: 51-59.

Gyngard, F. and Steinhauser, M.L. (2019) Biological explorations with nanoscale secondary ion mass spectrometry. Journal of Analytical Atomic Spectrometry 34: 1534-1545.

Hapca, S., Baveye, P.C., Wilson, C., Lark, R.M., and Otten, W. (2015) Three-dimensional mapping of soil chemical characteristics at micrometric scale by combining 2D SEMEDX data and 3D X-Ray CT images. PloS one 10:.

Hatzenpichler, R., Connon, S.A., Goudeau, D., Malmstrom, R.R., Woyke, T., and Orphan, V.J. (2016) Visualizing in situ translational activity for identifying and sorting slow-growing archaeal- bacterial consortia. Proceedings of the National Academy of Sciences 113: E4069-E4078. 
Hatzenpichler, R., Krukenberg, V., Spietz, R.L., and Jay, Z.J. (2020) Next-generation physiology approaches to study microbiome function at single cell level. Nature Reviews Microbiology 1-16.

Hatzenpichler, R. and Orphan, V.J. (2015) Detection of protein-synthesizing microorganisms in the environment via bioorthogonal noncanonical amino acid tagging (BONCAT). In Hydrocarbon and Lipid Microbiology Protocols. Springer, pp. 145-157.

Hatzenpichler, R., Scheller, S., Tavormina, P.L., Babin, B.M., Tirrell, D.A., and Orphan, V.J. (2014) In situ visualization of newly synthesized proteins in environmental microbes using amino acid tagging and click chemistry. Environmental microbiology 16: 25682590.

Hegermann, J., Wrede, C., Fassbender, S., Schliep, R., Ochs, M., Knudsen, L., and Mühlfeld, C. (2019) Volume-CLEM: a method for correlative light and electron microscopy in three dimensions. American Journal of Physiology-Lung Cellular and Molecular Physiology 317: L778-L784.

Hester, E.R., Harpenslager, S.F., van Diggelen, J.M., Lamers, L.L., Jetten, M.S., Lüke, C., et al. (2018) Linking nitrogen load to the structure and function of wetland soil and rhizosphere microbial communities. MSystems 3: e00214-17.

Hinz, F.I., Dieterich, D.C., Tirrell, D.A., and Schuman, E.M. (2011) Noncanonical amino acid labeling in vivo to visualize and affinity purify newly synthesized proteins in larval zebrafish. ACS chemical neuroscience 3: 40-49.

House, C.H., Cragg, B.A., and Teske, A. 2. DRILLING CONTAMINATION TESTS DURING ODP LEG 201 USING CHEMICAL AND PARTICULATE TRACERS.

Howarth, R.W. and Teal, J.M. (1979) Sulfate reduction in a New England salt marsh 1. Limnology and Oceanography 24: 999-1013.

Jochum, L.M., Schreiber, L., Marshall, I.P., Jørgensen, B.B., Schramm, A., and Kjeldsen, K.U. (2018) Single-cell genomics reveals a diverse metabolic potential of uncultivated Desulfatiglans-related Deltaproteobacteria widely distributed in marine sediment. Frontiers in microbiology 9: 2038.

Jörgensen, R.G., Raubuch, M., and Brandt, M. (2002) Soil microbial properties down the profile of a black earth buried by colluvium. Journal of plant nutrition and soil science $\mathbf{1 6 5}$ : 274-280.

Kawano, M. and Tomita, K. (2002) Microbiotic formation of silicate minerals in the weathering environment of a pyroclastic deposit. Clays and Clay Minerals 50: 99-110.

Knierim, B., Luef, B., Wilmes, P., Webb, R.I., Auer, M., Comolli, L.R., and Banfield, J.F. (2012) Correlative microscopy for phylogenetic and ultrastructural characterization of microbial communities. Environmental microbiology reports 4: 36-41.

Labonté, J.M., Lever, M.A., Edwards, K.J., and Orcutt, B.N. (2017) Influence of igneous basement on deep sediment microbial diversity on the eastern Juan de Fuca Ridge flank. Frontiers in microbiology 8: 1434.

LaRowe, D., Arndt, S., Bradley, J., Estes, E., Hoarfrost, A., Lang, S., et al. (2020) The fate of organic carbon in marine sediments-New insights from recent data and analysis. EarthScience Reviews 103146.

Larsen, S., Nielsen, L.P., and Schramm, A. (2015) Cable bacteria associated with long-distance electron transport in New England salt marsh sediment. Environmental microbiology reports 7: 175-179. 
Lehner, F., Kudlinzki, D., Richter, C., Müller-Werkmeister, H.M., Eberl, K.B., Bredenbeck, J., et al. (2017) Impact of azidohomoalanine incorporation on protein structure and ligand binding. ChemBioChem 18: 2340-2350.

Leizeaga, A., Estrany, M., Forn, I., and Sebastián, M. (2017) Using click-chemistry for visualizing in situ changes of translational activity in planktonic marine bacteria. Frontiers in microbiology 8: 2360.

Lovley, D.R. and Phillips, E.J. (1988) Novel mode of microbial energy metabolism: organic carbon oxidation coupled to dissimilatory reduction of iron or manganese. Appl Environ Microbiol 54: 1472-1480.

Lozupone, C.A. and Knight, R. (2007) Global patterns in bacterial diversity. Proceedings of the National Academy of Sciences 104: 11436-11440.

Lu, A., Li, Y., Jin, S., Wang, X., Wu, X.-L., Zeng, C., et al. (2012) Growth of non-phototrophic microorganisms using solar energy through mineral photocatalysis. Nature Communications 3: $1-8$.

Lucy, L.B. (1974) An iterative technique for the rectification of observed distributions. The astronomical journal 79: 745.

Mackey, K.R., Hunter-Cevera, K., Britten, G.L., Murphy, L.G., Sogin, M.L., and Huber, J.A. (2017) Seasonal succession and spatial patterns of Synechococcus microdiversity in a salt marsh estuary revealed through $16 \mathrm{~S}$ rRNA gene oligotyping. Frontiers in microbiology 8: 1496.

Mackin, J.E. and Swider, K.T. (1989) Organic matter decomposition pathways and oxygen consumption in coastal marine sediments. Journal of Marine Research 47: 681-716.

Marlow, J.J., Colocci, I., Jungbluth, S.P., Weber, N.M., Gartman, A., and Kallmeyer, J. (2020) Mapping metabolic activity at single cell resolution in intact volcanic fumarole sediment. FEMS Microbiology Letters 367: fnaa031.

Martin, M. (2011) Cutadapt removes adapter sequences from high-throughput sequencing reads. EMBnet journal 17: 10-12.

McGlynn, S.E., Chadwick, G.L., Kempes, C.P., and Orphan, V.J. (2015) Single cell activity reveals direct electron transfer in methanotrophic consortia. Nature 526: 531.

McGlynn, S.E., Chadwick, G.L., O’Neill, A., Mackey, M., Thor, A., Deerinck, T.J., et al. (2018) Subgroup Characteristics of Marine Methane-Oxidizing ANME-2 Archaea and Their Syntrophic Partners as Revealed by Integrated Multimodal Analytical Microscopy. Appl Environ Microbiol 84: e00399-18.

McMurdie, P.J. and Holmes, S. (2013) phyloseq: an R package for reproducible interactive analysis and graphics of microbiome census data. PloS one 8:

Mills, A.L., Herman, J.S., Hornberger, G.M., and DeJesús, T.H. (1994) Effect of solution ionic strength and iron coatings on mineral grains on the sorption of bacterial cells to quartz sand. Appl Environ Microbiol 60: 3300-3306.

Mitri, S., Clarke, E., and Foster, K.R. (2016) Resource limitation drives spatial organization in microbial groups. The ISME journal 10: 1471.

Morton, J.T., Sanders, J., Quinn, R.A., McDonald, D., Gonzalez, A., Vázquez-Baeza, Y., et al. (2017) Balance Trees Reveal Microbial Niche Differentiation. mSystems 2: e00162-16.

Musat, N., Musat, F., Weber, P.K., and Pett-Ridge, J. (2016) Tracking microbial interactions with NanoSIMS. Current opinion in biotechnology 41: 114-121.

Myers, C.R. and Nealson, K.H. (1988) Bacterial manganese reduction and growth with manganese oxide as the sole electron acceptor. Science 240: 1319-1321. 
Oremland, R.S. and Polcin, S. (1982) Methanogenesis and sulfate reduction: competitive and noncompetitive substrates in estuarine sediments. Appl Environ Microbiol 44: 12701276.

Parada, A.E., Needham, D.M., and Fuhrman, J.A. (2016) Every base matters: assessing small subunit rRNA primers for marine microbiomes with mock communities, time series and global field samples. Environmental microbiology 18: 1403-1414.

Pasulka, A.L., Thamatrakoln, K., Kopf, S.H., Guan, Y., Poulos, B., Moradian, A., et al. (2018) Interrogating marine virus-host interactions and elemental transfer with BONCAT and nanoSIMS-based methods. Environmental microbiology 20: 671-692.

Paulson, J.N., Pop, M., and Bravo, H.C. (2013) metagenomeSeq: Statistical analysis for sparse high-throughput sequencing. Bioconductor package 1: 191.

Pausan, M.R., Csorba, C., Singer, G., Till, H., Schöpf, V., Santigli, E., et al. (2019) Exploring the archaeome: detection of archaeal signatures in the human body. Frontiers in Microbiology 10: 2796.

Peng, X., Yando, E., Hildebrand, E., Dwyer, C., Kearney, A., Waciega, A., et al. (2013) Differential responses of ammonia-oxidizing archaea and bacteria to long-term fertilization in a New England salt marsh. Frontiers in microbiology 3: 445.

Pohlner, M., Dlugosch, L., Wemheuer, B., Mills, H., Engelen, B., and Reese, B.K. (2019) The Majority of Active Rhodobacteraceae in Marine Sediments Belong to Uncultured Genera: A Molecular Approach to Link Their Distribution to Environmental Conditions. Frontiers in Microbiology 10: 659.

Pujalte, M.J., Lucena, T., Ruvira, M.A., Arahal, D.R., and Macián, M.C. (2014) The family rhodobacteraceae. Springer.

Quast, C., Pruesse, E., Yilmaz, P., Gerken, J., Schweer, T., Yarza, P., et al. (2012) The SILVA ribosomal RNA gene database project: improved data processing and web-based tools. Nucleic acids research 41: D590-D596.

Reichart, N.J., Jay, Z.J., Krukenberg, V., Parker, A.E., Spietz, R.L., and Hatzenpichler, R. (2020) Activity-based cell sorting reveals responses of uncultured archaea and bacteria to substrate amendment. The ISME $J$ accepted:

Richardson, W.H. (1972) Bayesian-based iterative method of image restoration. JOSA 62: 5559.

Rogers, J.R. and Bennett, P.C. (2004) Mineral stimulation of subsurface microorganisms: release of limiting nutrients from silicates. Chemical Geology 203: 91-108.

Romine, M.F., Rodionov, D.A., Maezato, Y., Osterman, A.L., and Nelson, W.C. (2017) Underlying mechanisms for syntrophic metabolism of essential enzyme cofactors in microbial communities. The ISME journal 11: 1434.

Salman, V., Yang, T., Berben, T., Klein, F., Angert, E., and Teske, A. (2015) Calciteaccumulating large sulfur bacteria of the genus Achromatium in Sippewissett Salt Marsh. The ISME journal 9: 2503-2514.

Samo, T.J., Smriga, S., Malfatti, F., Sherwood, B.P., and Azam, F. (2014) Broad distribution and high proportion of protein synthesis active marine bacteria revealed by click chemistry at the single cell level. Frontiers in Marine Science 1: 48.

Schindelin, J., Arganda-Carreras, I., Frise, E., Kaynig, V., Longair, M., Pietzsch, T., et al. (2012) Fiji: an open-source platform for biological-image analysis. Nature methods 9: 676-682.

Schlesner, H., Rensmann, C., Tindall, B.J., Gade, D., Rabus, R., Pfeiffer, S., and Hirsch, P. (2004) Taxonomic heterogeneity within the Planctomycetales as derived by DNA-DNA 
hybridization, description of Rhodopirellula baltica gen. nov., sp. nov., transfer of Pirellula marina to the genus Blastopirellula gen. nov. as Blastopirellula marina comb. nov. and emended description of the genus Pirellula. International journal of systematic and evolutionary microbiology 54: 1567-1580.

Schlüter, S., Eickhorst, T., and Mueller, C.W. (2018) Correlative imaging reveals holistic view of soil microenvironments. Environmental science \& technology 53: 829-837.

Sebastián, M., Estrany, M., Ruiz-González, C., Forn, I., Sala, M.M., Gasol, J.M., and Marrasé, C. (2019) High growth potential of long-term starved deep ocean opportunistic heterotrophic bacteria. Frontiers in microbiology 10: 760.

Seitz, A.P., Nielsen, T.H., and Overmann, J. (1993) Physiology of purple sulfur bacteria forming macroscopic aggregates in Great Sippewissett Salt Marsh, Massachusetts. FEMS microbiology ecology 12: 225-235.

Shapiro, O.H., Hatzenpichler, R., Buckley, D.H., Zinder, S.H., and Orphan, V.J. (2011) Multicellular photo-magnetotactic bacteria. Environmental microbiology reports 3: 233238.

Sharma, K., Palatinszky, M., Nikolov, G., Berry, D., and Shank, E.A. (In Press) Transparent soil microcosms enable real-time imaging and non-destructive stable isotope probing of bacteria and fungi. eLife.

Shelobolina, E., Xu, H., Konishi, H., Kukkadapu, R., Wu, T., Blöthe, M., and Roden, E. (2012) Microbial lithotrophic oxidation of structural Fe (II) in biotite. Appl Environ Microbiol 78: 5746-5752.

Shi, L., Dong, H., Reguera, G., Beyenal, H., Lu, A., Liu, J., et al. (2016) Extracellular electron transfer mechanisms between microorganisms and minerals. Nature Reviews Microbiology 14: 651.

Sletten, E.M. and Bertozzi, C.R. (2009) Bioorthogonal chemistry: fishing for selectivity in a sea of functionality. Angewandte Chemie International Edition 48: 6974-6998.

Smith, D.C., Spivack, A.J., Fisk, M.R., Haveman, S.A., Staudigel, H., and Party, L. (2000) Methods for quantifying potential microbial contamination during deep ocean coring. Ocean Drilling Program Technical Note.

Spain, A.M., Krumholz, L.R., and Elshahed, M.S. (2009) Abundance, composition, diversity and novelty of soil Proteobacteria. The ISME journal 3: 992-1000.

Spaink, H.P., Kondorosi, A., and Hooykaas, P.J. (2012) The Rhizobiaceae: molecular biology of model plant-associated bacteria, Springer Science \& Business Media.

Spring, S., Fiebig, A., Riedel, T., Göker, M., and Klenk, H.-P. (2014) Genome sequence of gammaproteobacterial Pseudohaliea rubra type strain DSM 19751, isolated from coastal seawater of the Mediterranean Sea. Genome Announc 2: e01208-14.

Spring, S., Scheuner, C., Göker, M., and Klenk, H.-P. (2015) A taxonomic framework for emerging groups of ecologically important marine gammaproteobacteria based on the reconstruction of evolutionary relationships using genome-scale data. Frontiers in microbiology 6: 281.

Steward, K.F., Eilers, B., Tripet, B., Fuchs, A., Dorle, M., Rawle, R., et al. (2020) Metabolic Implications of Using BioOrthogonal Non-Canonical Amino Acid Tagging (BONCAT) for Tracking Protein Synthesis. Frontiers in Microbiology 11: 197.

Stone, M., DeForest, J., and Plante, A. (2014) Changes in extracellular enzyme activity and microbial community structure with soil depth at the Luquillo Critical Zone Observatory. Soil Biology and Biochemistry 75: 237-247. 
1002

1003

1004

1005

1006

1007

1008

1009

1010

1011

1012

1013

1014

1015

1016

1017

1018

1019

1020

1021

1022

1023

1024

Thamdrup, B. (2000) Bacterial manganese and iron reduction in aquatic sediments. In Advances in microbial ecology. Springer, pp. 41-84.

Thompson, L.R., Sanders, J.G., McDonald, D., Amir, A., Ladau, J., Locey, K.J., et al. (2017) A communal catalogue reveals Earth's multiscale microbial diversity. Nature 551:

Tobias, C. and Neubauer, S.C. (2019) Salt marsh biogeochemistry-an overview. In Coastal wetlands. Elsevier, pp. 539-596.

Valsami-Jones, E., McLean, J., McEldowney, S., Hinrichs, H., and Pili, A. (1998) An experimental study of bacterially induced dissolution of K-feldspar. Mineral Mag A 62 : $1563-1564$.

Welch, S.A. and Banfield, J.F. (2002) Modification of olivine surface morphology and reactivity by microbial activity during chemical weathering. Geochimica et Cosmochimica Acta 66: 213-221.

West, S.A., Diggle, S.P., Buckling, A., Gardner, A., and Griffin, A.S. (2007) The social lives of microbes. Annu Rev Ecol Evol Syst 38: 53-77.

Wilbanks, E.G., Jaekel, U., Salman, V., Humphrey, P.T., Eisen, J.A., Facciotti, M.T., et al. (2014) Microscale sulfur cycling in the phototrophic pink berry consortia of the Sippewissett Salt Marsh. Environmental microbiology 16: 3398-3415.

Wilbanks, E.G., Salman-Carvalho, V., Jaekel, U., Humphrey, P.T., Eisen, J.A., Buckley, D.H., and Zinder, S.H. (2017) The green berry consortia of the Sippewissett salt marsh: Millimeter-sized aggregates of diazotrophic unicellular cyanobacteria. Frontiers in microbiology 8: 1623.

Wittebolle, L., Marzorati, M., Clement, L., Balloi, A., Daffonchio, D., Heylen, K., et al. (2009) Initial community evenness favours functionality under selective stress. Nature 458: 623626.

Wright, J.J., Konwar, K.M., and Hallam, S.J. (2012) Microbial ecology of expanding oxygen minimum zones. Nature Reviews Microbiology 10: 381.

Ye, R., Jin, Q., Bohannan, B., Keller, J.K., and Bridgham, S.D. (2014) Homoacetogenesis: A potentially underappreciated carbon pathway in peatlands. Soil Biology and Biochemistry 68: 385-391.

Ye, S., Zhang, Y., and Sleep, B.E. (2015) Distribution of biofilm thickness in porous media and implications for permeability models. Hydrogeology journal 23: 1695-1702.

Zhou, S., Tang, J., Yuan, Y., Yang, G., and Xing, B. (2018) TiO2 Nanoparticle-Induced Nanowire Formation Facilitates Extracellular Electron Transfer. Environ Sci Technol Lett 5: 564-570.

\section{Figure and Table Captions}

Fig. 1: Correlative fluorescence and electron microscopy from the uppermost section (7.6 mm sediment depth). 1) Overlain on the base SEM image are two fluorescence channels showing SYBR-active features in blue, and BONCAT-active features in yellow. The dark zonation indicates the fluorescence microscopy footprint. 2), 3), and 4) show three mineralogically distinct 
1029 sites in detail. i) SYBR green, ii) BONCAT, and iii) merged channels, as well as iv) EDS elemental 1030 abundance maps (in which dark blue background represents the resin).

1031 Fig 2: Histograms of the relative proportions of all organisms and the anabolically active 1032 subset (yellow overlay) at given distances from the mineral surface for the uppermost section (7.6 1033 mm sediment depth). 1) Data for all grains. 2) Data separated by mineral type. Histogram bins are

1034 in $5 \mu \mathrm{m}$ intervals, and only cells located outside mineral surfaces are shown. 3) Composite 1035 elemental maps derived from EDS analysis show the mineral grains that were analyzed, labeled 1036 by mineral type. $\mathrm{A}=$ quartz; $\mathrm{B}=$ Plagioclase; $\mathrm{C}=$ Orthoclase; $\mathrm{D}=$ Rutile; $\mathrm{E}=$ Albite; $\mathrm{F}=$ 1037 Ca,K,Mg,Fe silicate; $\mathrm{G}=$ Hornblende.

1038 Fig. 3: Correlative fluorescence and electron microscopy from the embedded section at 12 1039 mm sediment depth. 1) Overlain on the base SEM image are two fluorescence channels showing 1040 SYBR-active features in blue, and BONCAT-active features in yellow. The dark zonation 1041 indicates the fluorescence microscopy footprint. 2), 3), and 4) show three mineralogically distinct 1042 sites in additional detail in i) SYBR green, ii) BONCAT, and iii) merged channels, as well as iv) 1043 EDS elemental abundance maps (in which dark blue background represents the resin).

1044 Fig 4: Histograms of the relative proportions of all organisms and the anabolically active 1045 subset (yellow overlay) at given distances from the mineral surface for the middle analyzed section 1046 (12 mm sediment depth). 1) Data for all grains. 2) Data separated by mineral type. Histogram bins 1047 are in $5 \mu \mathrm{m}$ intervals, and only cells located outside mineral surfaces are shown. 3) Composite 1048 elemental maps derived from EDS analysis show the mineral grains that were analyzed, labeled 1049 by mineral type. $\mathrm{A}=$ quartz; $\mathrm{B}=$ Plagioclase; $\mathrm{C}=$ Orthoclase; $\mathrm{D}=$ Rutile; $\mathrm{E}=$ Albite; $\mathrm{F}=$ $1050 \mathrm{Ca}, \mathrm{K}, \mathrm{Mg}$, Fe silicate; $\mathrm{G}=$ Hornblende. 
Fig. 5: Trends of the relative abundances of active and inactive subsets of the eight most prevalent orders with sediment depth, as detected by BONCAT-FACS combined with 16S rRNA

1053 gene sequencing $(\mathrm{n}=3)$. At each horizon, the relative abundance contribution for each order was

1054 determined in both the anabolically active sorted cells and the inactive sorted cells. Values to the 1055 right of the axis indicate the relative abundance of that order in the active fraction; values to the 1056 left indicate the relative abundance in the inactive fraction. The colored bars reveal if the order 1057 was enriched in the active fraction (yellow bars) or the inactive fraction (blue bars) in a given 1058 horizon. The length of bars show fold-enrichment, as indicated by the x-axis, calculated by 1059 dividing the larger relative abundance value by the smaller relative abundance value for each 1060 horizon.

Fig. 6: Correlative fluorescence and electron microscopy from the embedded section at $106260.7 \mathrm{~mm}$ sediment depth. 1) Overlain on the base SEM image are two fluorescence channels 1063 showing SYBR-active features in blue, and BONCAT-active features in yellow. The dark zonation 1064 indicates the fluorescence microscopy footprint. 2), 3), and 4) show three mineralogically distinct 1065 sites in additional detail in i) SYBR green, ii) BONCAT, and iii) merged channels, as well as iv) 1066 EDS elemental abundance maps (in which dark blue background represents the resin).

1068 subset (yellow overlay) at given distances from the mineral surface for the lowest analyzed section 1069 (60.7 mm sediment depth). 1) Data for all grains. 2) Data separated by mineral type. Histogram 1070 bins are in $5 \mu \mathrm{m}$ intervals, and only cells located outside mineral surfaces are shown. 3) Composite 1071 elemental maps derived from EDS analysis show the mineral grains that were analyzed, labeled 1072 by mineral type. $\mathrm{A}=$ quartz; $\mathrm{B}=$ Plagioclase; $\mathrm{C}=$ Orthoclase; $\mathrm{D}=$ Rutile; $\mathrm{E}=$ Albite; $\mathrm{F}=$ $1073 \mathrm{Ca}, \mathrm{K}, \mathrm{Mg}$,Fe silicate; $\mathrm{G}=$ Hornblende. 
Fig. 8: An overview of the experimental and sample processing approach deployed in this

1075 study. The PETG tube is cut to the appropriate dimensions and the lower edge is beveled (1). Cut-

1076 off $50 \mathrm{~mL}$ Falcon tube tops are secured to the PETG tube with epoxy (2), and sediment is collected

1077 from the marsh by pressing the tube downward into the sediment (3). A sterile plug of glass wool

1078 is added to the bottom to keep the material in place, and the full tube is pulled back out of the

1079 marsh. Tube lids are secured; the top lid has a perforated top to allow contact with an oxic

1080 atmosphere (4). In an anoxic chamber, lids are removed and fluid is replaced drop-wise by pipette

1081 with $50 \mu \mathrm{M}$ HPG in $0.22 \mu \mathrm{m}$-filtered Berry Pool water (5). (Not all cycles of fluid replacement are

1082 shown; see text for full protocol.) PDMS membranes are secured to top and bottom of tube with

1083 twist-on lids (6). Sample tubes are returned to the marsh; immediately prior to emplacement in the

1084 Berry Pool sediment, the bottom lid is perforated to allow gaseous continuity with the environment

1085 (7). The sample is placed back in the sediment at the initial collection location for the duration of

1086 the incubation period (8); upon recovery, lid perforations are immediately covered with electrical

1087 tape to minimize gas exchange during transport back to the lab (9). In the anoxic chamber, 1088 incubation fluid is replaced with fixative and incubated for four hours at room temperature (10).

1089 Correlative microscopy cores are processed according to steps 11a-15a. The fixed core is

1090 removed from the anoxic chamber and infiltrated with an ethanol dehydration series (11a) followed

1091 by LR White resin (12a), which is allowed to cure during a 36 hour incubation at $60{ }^{\circ} \mathrm{C}$. The

1092 embedded core is then sectioned by sterile water-cooled diamond saw (13a), and sectioned

1093 surfaces are incubated in the click solution for 60 minutes in the dark in an anoxic chamber (14a).

1094 Sample sections are now ready for SYBR green counterstaining and fluorescence and electron 1095 microscopy (15a). 
Cores for cell sorting and sequencing are processed according to steps $11 \mathrm{~b}-15 \mathrm{~b}$. The

1097 overlying liquid and top $1.0 \mathrm{~cm}$ of sediment is removed and replaced by a plug of sterile glass

1098 wool for transport (11b). A sterile plunger was used to extrude the core in $1 \mathrm{~cm}$ increments (12b).

1099 Cells were extracted from these subsamples and then incubated in the click solution for 30 minutes

1100 in the dark (13b). Cells were then washed (14b) and introduced to the cell sorter, which separated

1101 BONCAT positive and BONCAT negative cells (15b) for downstream sequencing.

1102 Table 1: Cell abundance and percentage of anabolically active cells as determined through

1103 fluorescence microscopy and BONCAT-FACS analyses.

1104 Table 2: Proportions of cells, and the anabolically active subsets, associated with mineral

1105 exteriors and interiors at the three horizons examined by correlative microscopy. For the biomass

1106 per surface area and volume, the relative proportion of biomass associated with a given mineral

1107 type was divided by the relative proportion of surface area or volume accounted for by that mineral

1108 type. Values less than 1 indicate fewer associated cells than would be expected given an even

1109 distribution of biomass across mineral perimeters or surfaces. Only mineral types that accounted

1110 for at least 5\% of the observed biomass in a given horizon are included in this analysis.

\section{Supplemental Figure and Table Captions}

1112 Fig. S1: Sampling site at Little Sippewissett salt marsh. 1) A satellite image of the marsh

1113 acquired on October 6 ${ }^{\text {th }}, 2018$ (Google Earth). The white box indicates the "Berry Pool" shown in

1114 image 2 where the sampling was conducted. The white arrow indicates the direction in which

1115 image 2 was acquired. 2) The "Berry Pool", so named because of its high abundance of

1116 phototrophic pink berries, as seen on August $25^{\text {th }}, 2018$. The white arrow points to the site of 1117 sample acquisition on September $\left.26^{\text {th }}, 2018.3\right)$ A closer view of the sediment surface at low tide 
1118 on September $26^{\text {th }}$, showing pink berries, organic surface cover, and sandy sediment. 4) Custom-

1119 built sample chambers placed at the site of collection for incubation.

1120 Fig. S2: FACS plots. (A) Shows the no HPG controls used to draw gates around the

1121 BONCAT positive (active) and BONCAT negative (inactive) cell fractions in the HPG-added

1122 sediment core (B). Note that the biomass extracted from the no HPG control (A) was much lower

1123 than seen in other samples where HPG was added.

1124 Fig. S3: Alpha diversity metrics of the bulk, BONCAT+ (Cy5+), and BONCAT- (Cy5-)

1125 communities analyzed by 16S rRNA gene amplicon sequencing for each sediment horizon.

$1126 \quad$ Fig. S4: Beta diversity metrics derived from 16S rRNA gene amplicon sequence data. 1)

1127 NMDS comparing bulk community with active/inactive sorted communities (stress 0.0784916).

1128 2) NMDS showing differences in sorted active/inactive communities by depth (stress 0.1517008$)$.

1129 Fig. S5: Representative fields of view of E. coli cultures exposed to different concentrations

1130 of HPG and the azide dye. The $50 \mu \mathrm{M}$ HPG, $5 \mu \mathrm{M}$ dye combination provided the best combination

1131 of coverage and dynamic range and was used in the field-based experimental incubation. All

1132 samples were stained with the general DNA stain DAPI.

1133 Fig. S6: The percentage of Sippewissett biomass that was anabolically active as a function

1134 of incubation time. All incubations used homogenized LSSM sediment from the 0-5 cm horizon,

1135 and received $50 \mu \mathrm{M}$ HPG. Active and inactive organisms were quantified as described in the text.

1136 Data points represent mean and standard deviation values across 5 fields of view; one field of view

1137 is provided in both SYBR green and BONCAT channels for 1, 44, and 88 hours below the graph.

1138 Fig. S7: To co-register fluorescence and electron microscopy images and facilitate precise

1139 spatial analysis, the bUnwarpJ algorithm in FIJI / ImageJ was used. See the text for details on

1140 parameter settings. 
Fig. S8: To determine the z-axis depth into the embedded section that our protocol would

1142 detect, Cy3 (BONCAT) and SYBR green (all cells) channels were recorded at multiple focal

1143 depths with a step size of $0.35 \mu \mathrm{m}$. Three BONCAT and two SYBR green features are highlighted.

1144 Each resulting image was processed identically, as described in the text; Cy3 signal was color-

1145 shifted to yellow and SYBR green signal was color-shifted to blue for viewing ease. Features that

1146 registered as an "object" after processing have a yellow or blue border; those that were not have

1147 no border. For each feature, the depth-based analysis began when the object was fully in focus.

1148 Fig. S9: Histograms of the number of organisms as a function of distance from mineral

1149 surfaces. Histogram bins are in increments of $5 \mu \mathrm{m}$; the number of each bin corresponds to the

1150 upper bound of the range (e.g., " 5 " includes all organisms between 0 and $5 \mu \mathrm{m}$ from the mineral

1151 surface). Only organisms on the outside of mineral grains are shown. Mineral types with less than

115283 associated cells (e.g., $<1 \%$ of the total mineral-external cells observed in this study) are not

1153 shown.

1154 Fig. S10: To test the effect of our fluid replacement approach on transport of microbial

1155 cells, 1-micron fluorescent beads were introduced to the overlying water and tracked through the

1156 core during the fluid replacement and embedding process. Horizons of fluorescent bead

1157 quantification are indicated by green arrows, and representative fields of view are shown at right.

1158 Horizons analyzed for correlative microscopy and FACS-16S rRNA gene sequencing are shown

1159 with black arrows and blue brackets, respectively.

1160 Table S1: Details on the conditions and analyses to which experimental and control 1161 sediment cores were subjected. 


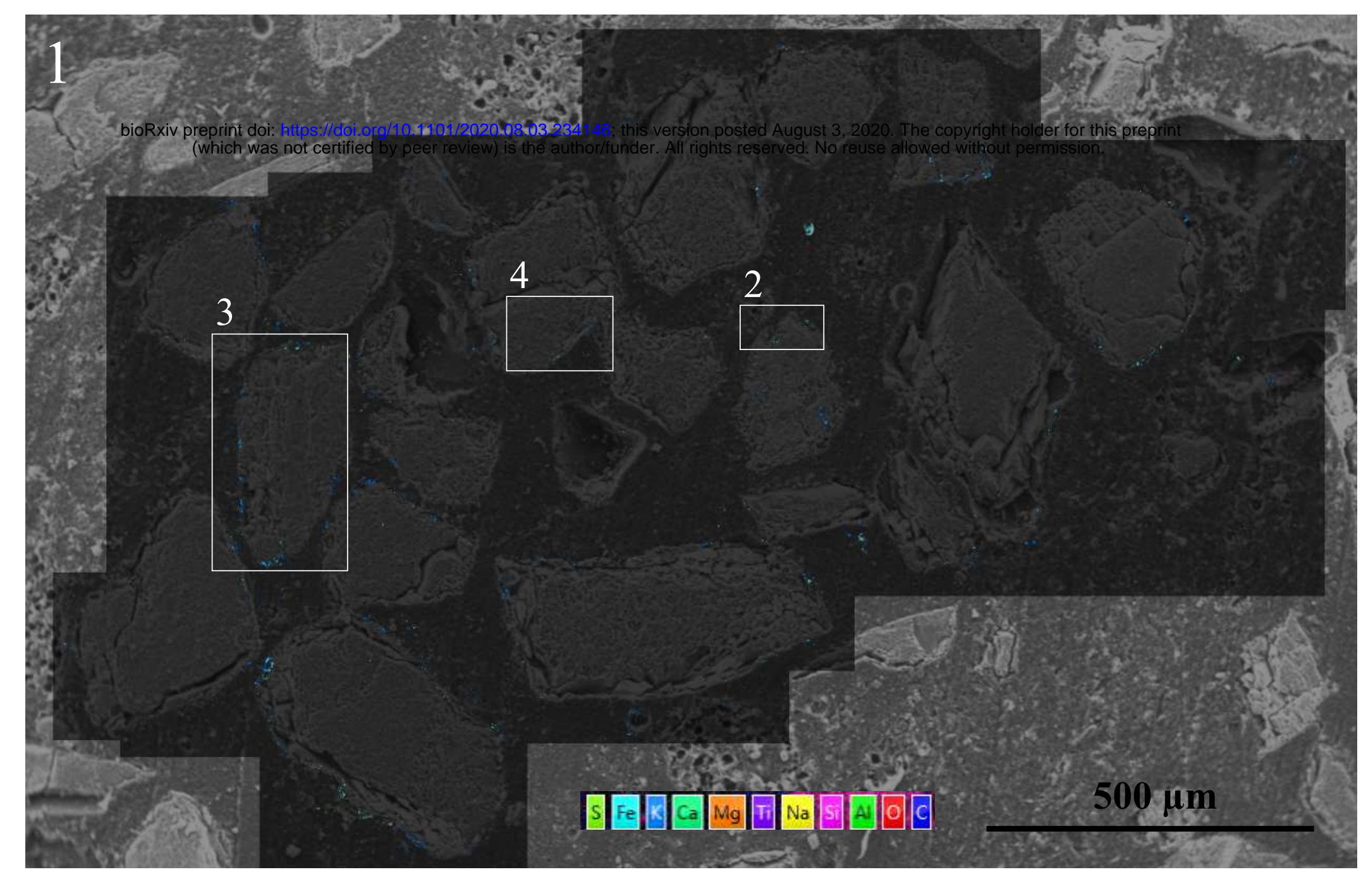

\section{2: Rutile}

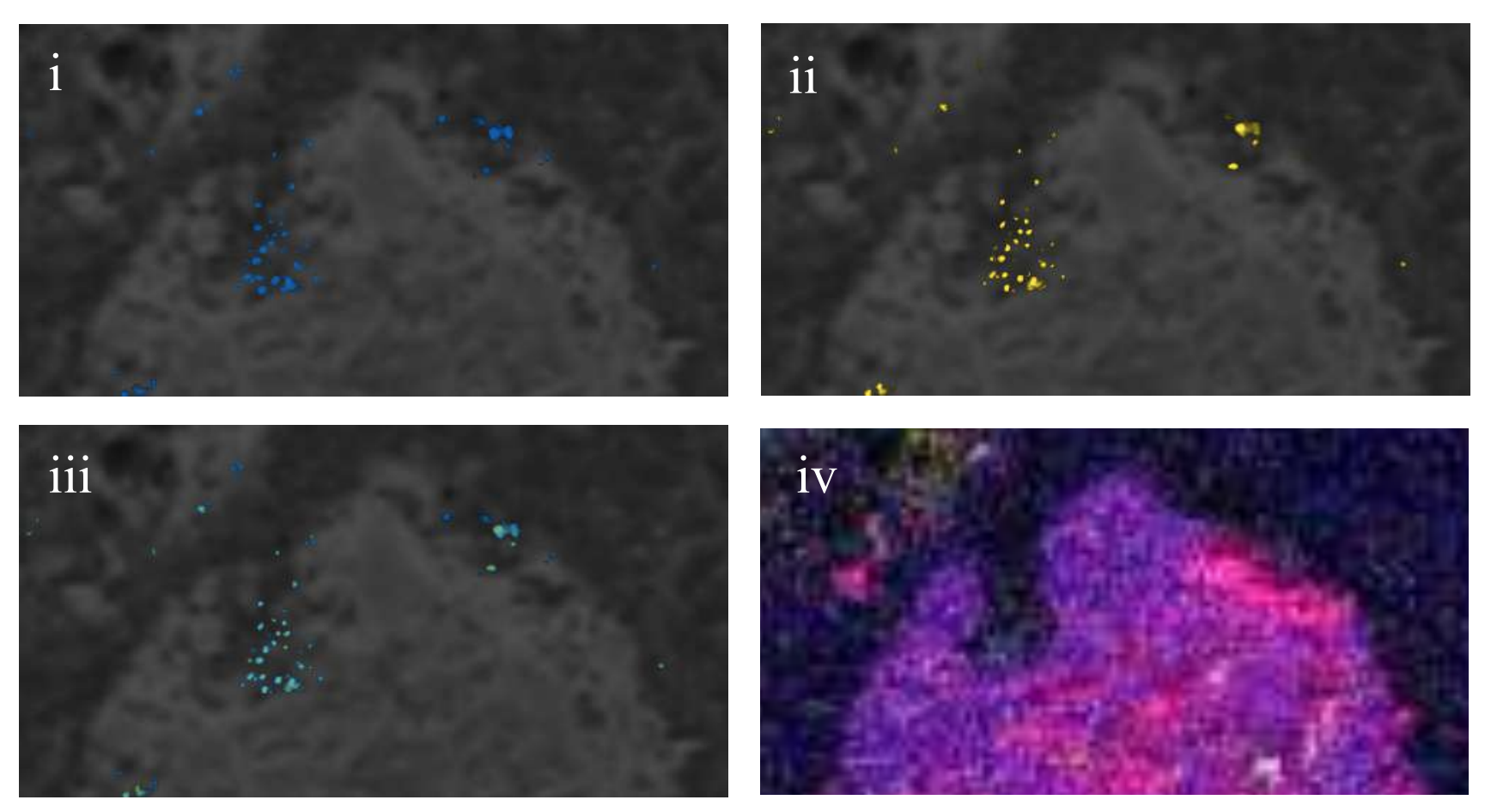

3: Plagioclase

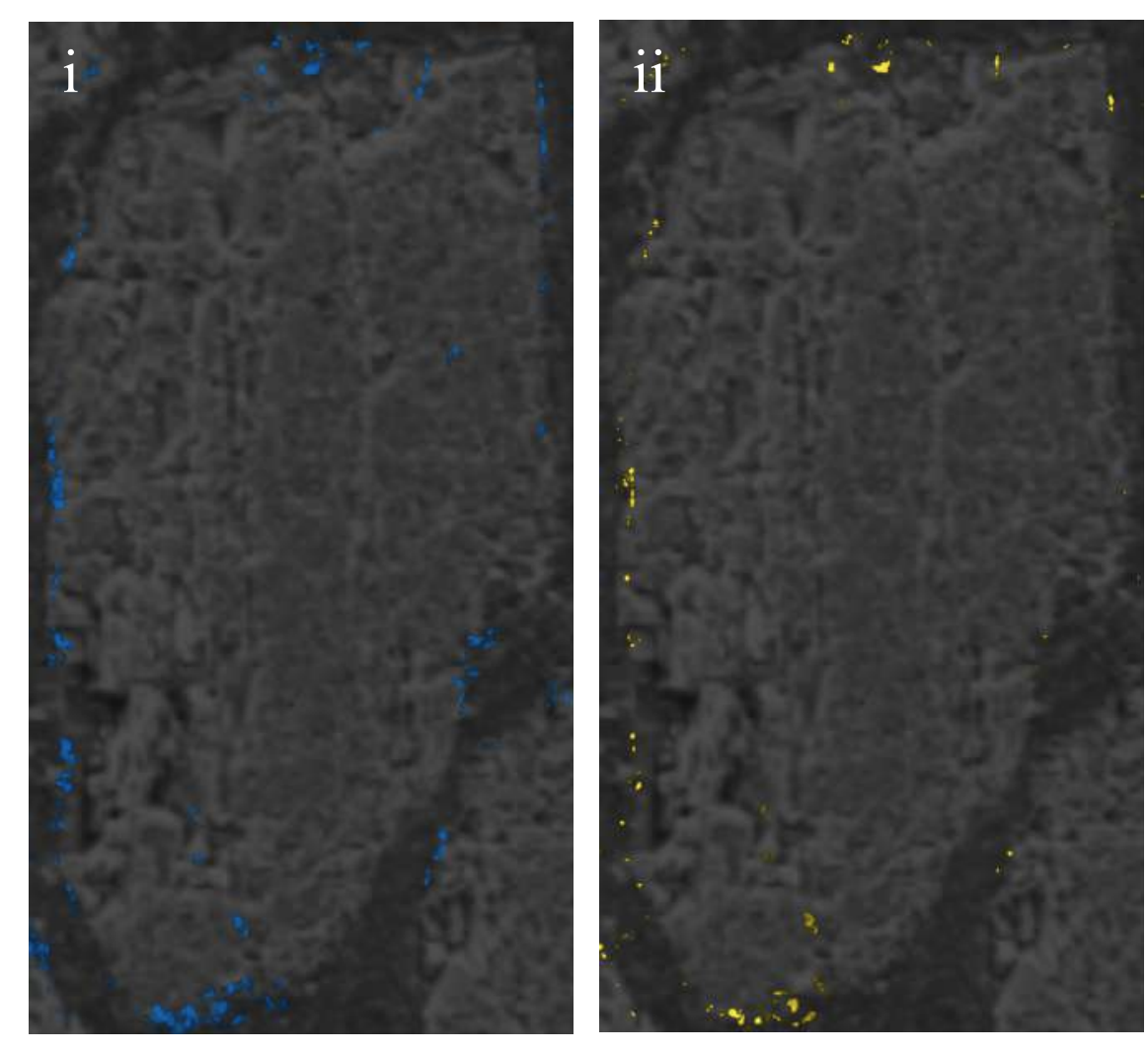

4: Quartz

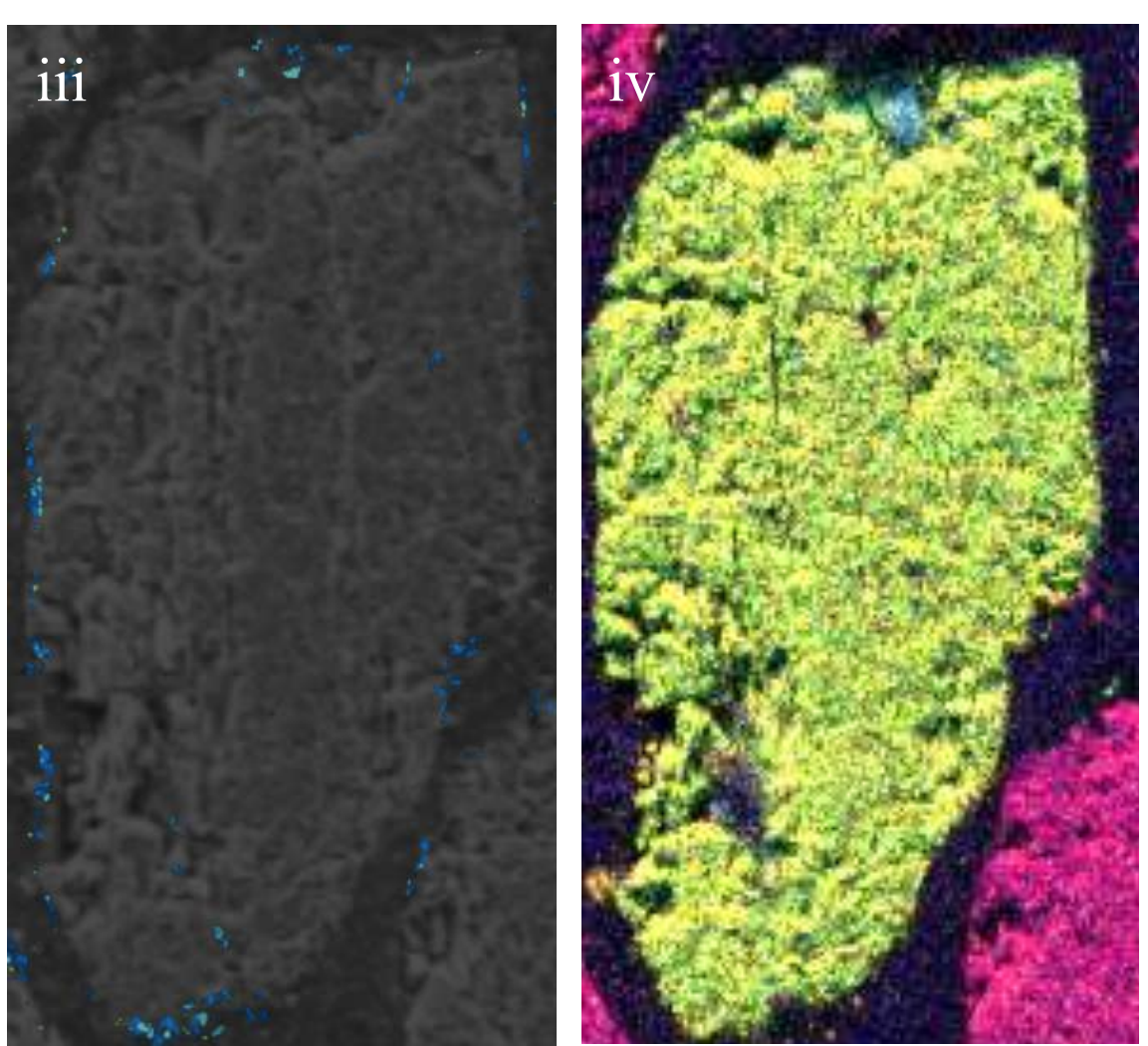

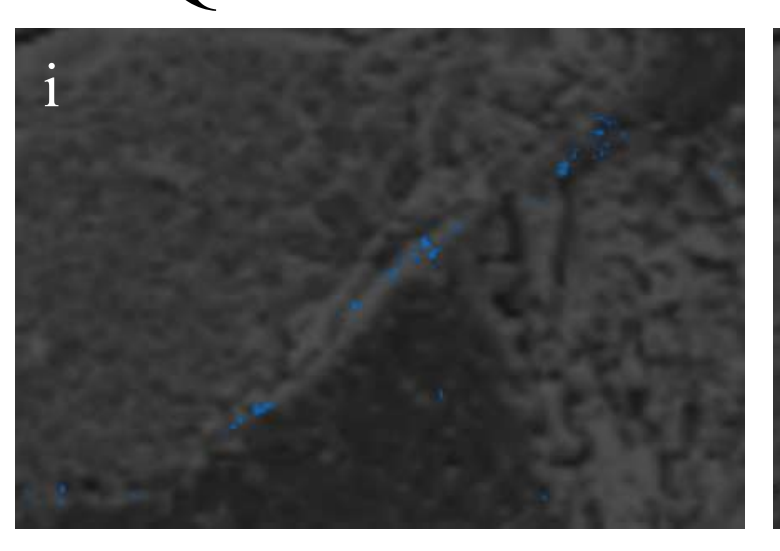

iii
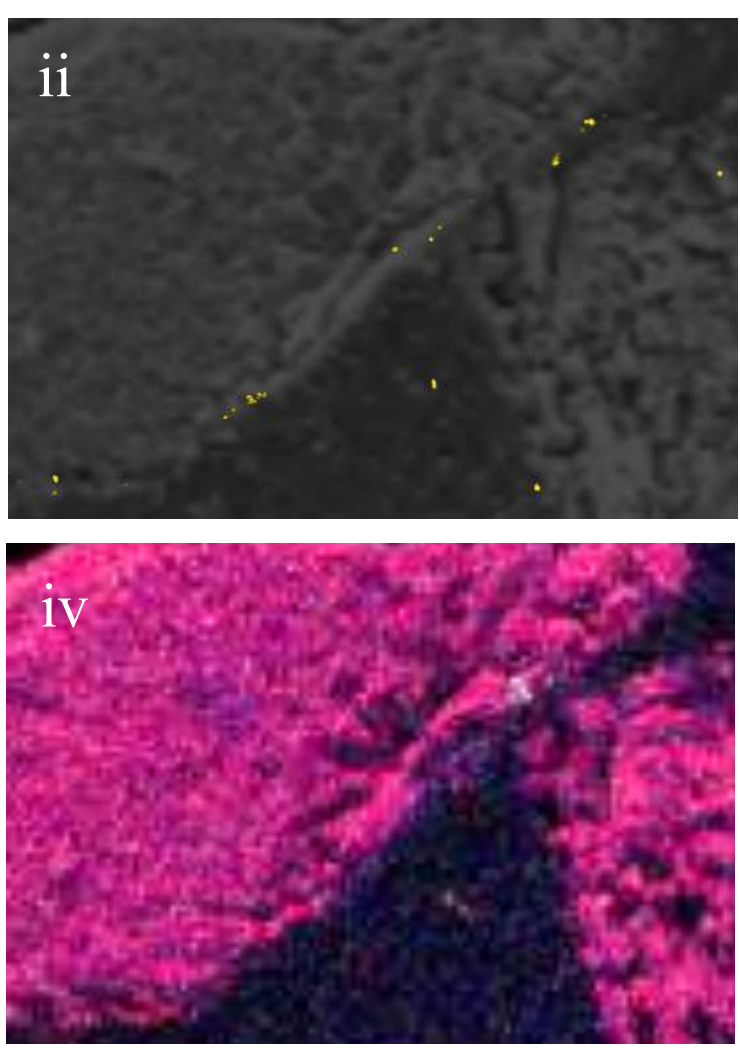


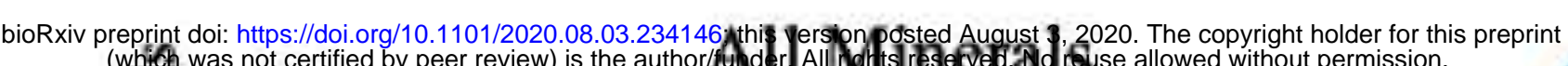

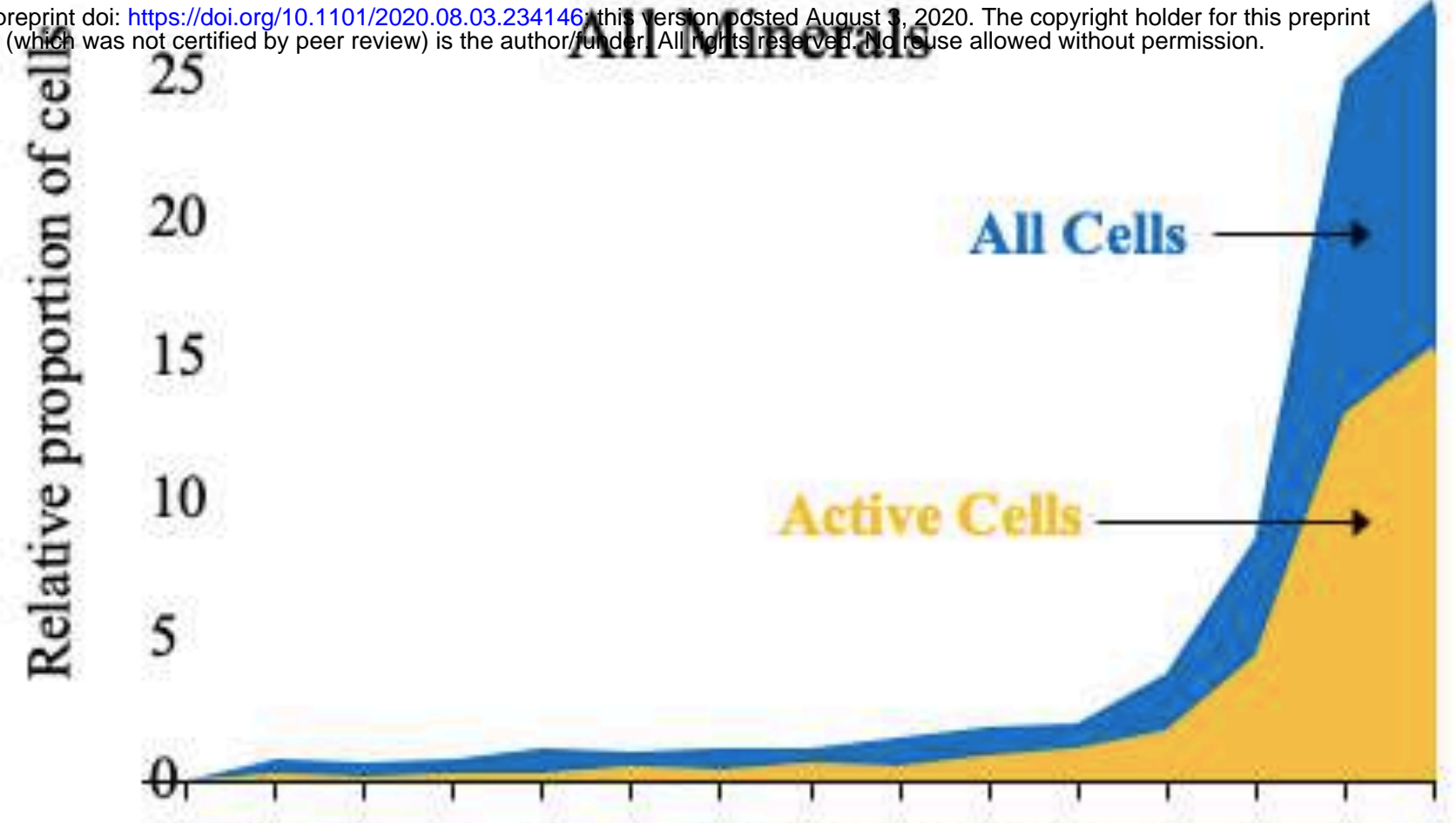

$\begin{array}{lllllllllllllll}70 & 65 & 60 & 55 & 50 & 45 & 40 & 35 & 30 & 25 & 20 & 15 & 10 & 5 & 0\end{array}$

Distance from Mineral Surface $(\mu \mathrm{m})$

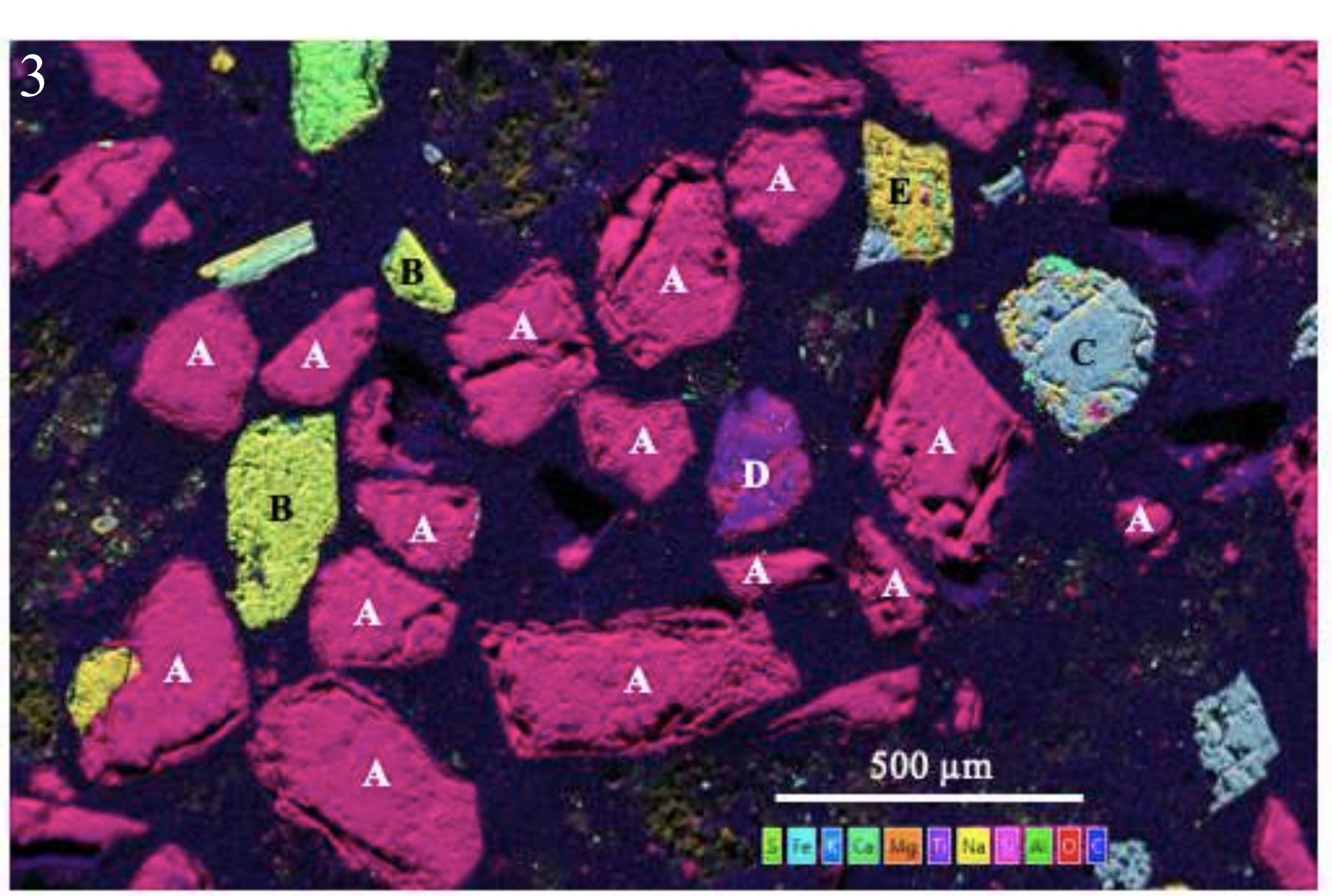

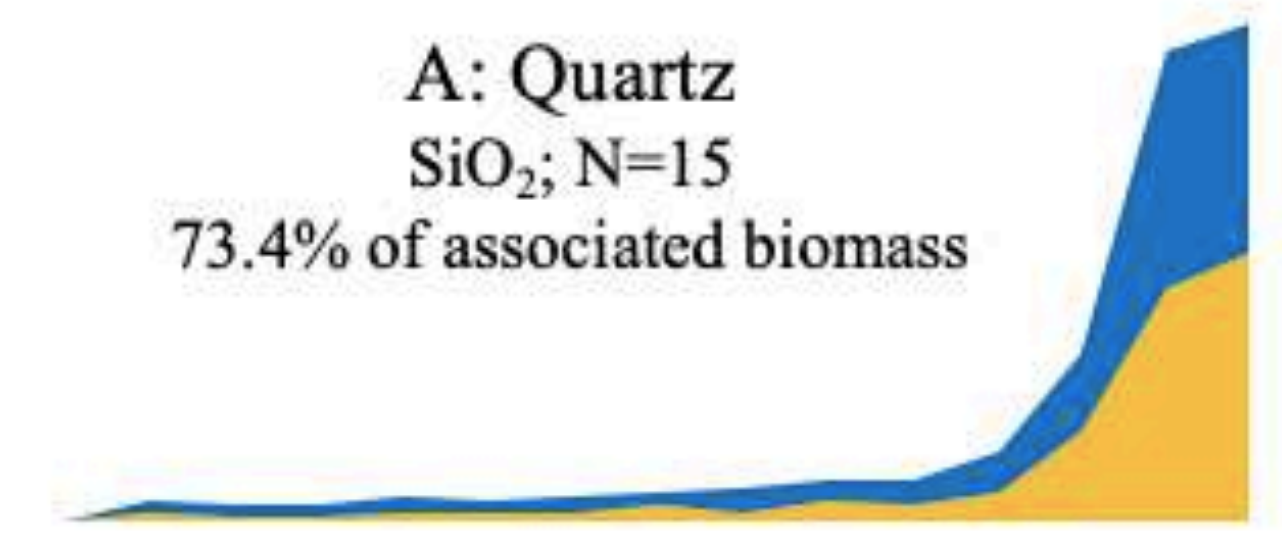

잉

B: Plagioclase Feldspar $\mathrm{NaAlSi}_{3} \mathrm{O}_{8}-\mathrm{CaAl}_{2} \mathrm{Si}_{2} \mathrm{O}_{8} ; \mathrm{N}=2$

$10 \%$ of associated biomass

10

…

.

40 


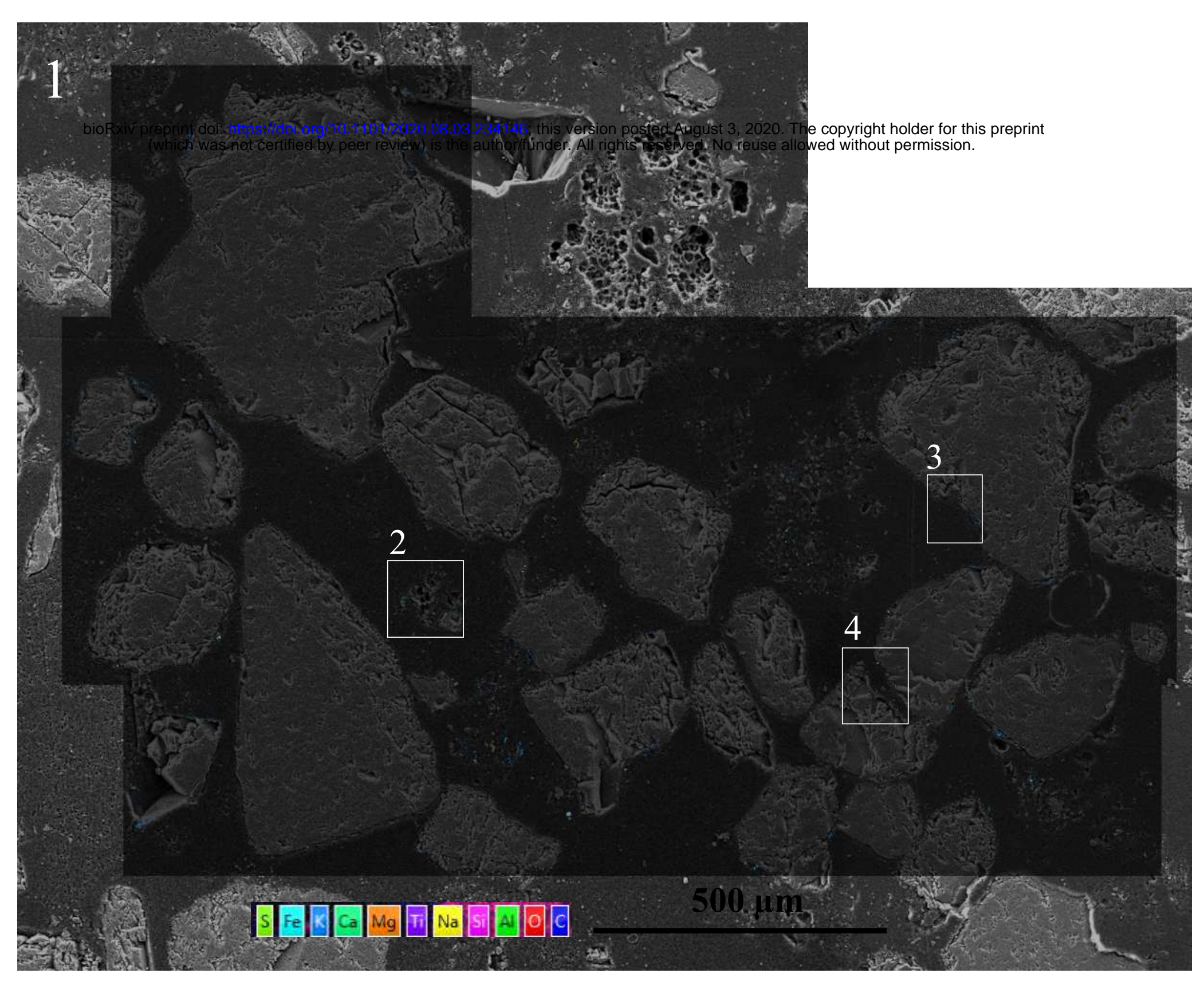

2: Particle Assemblage
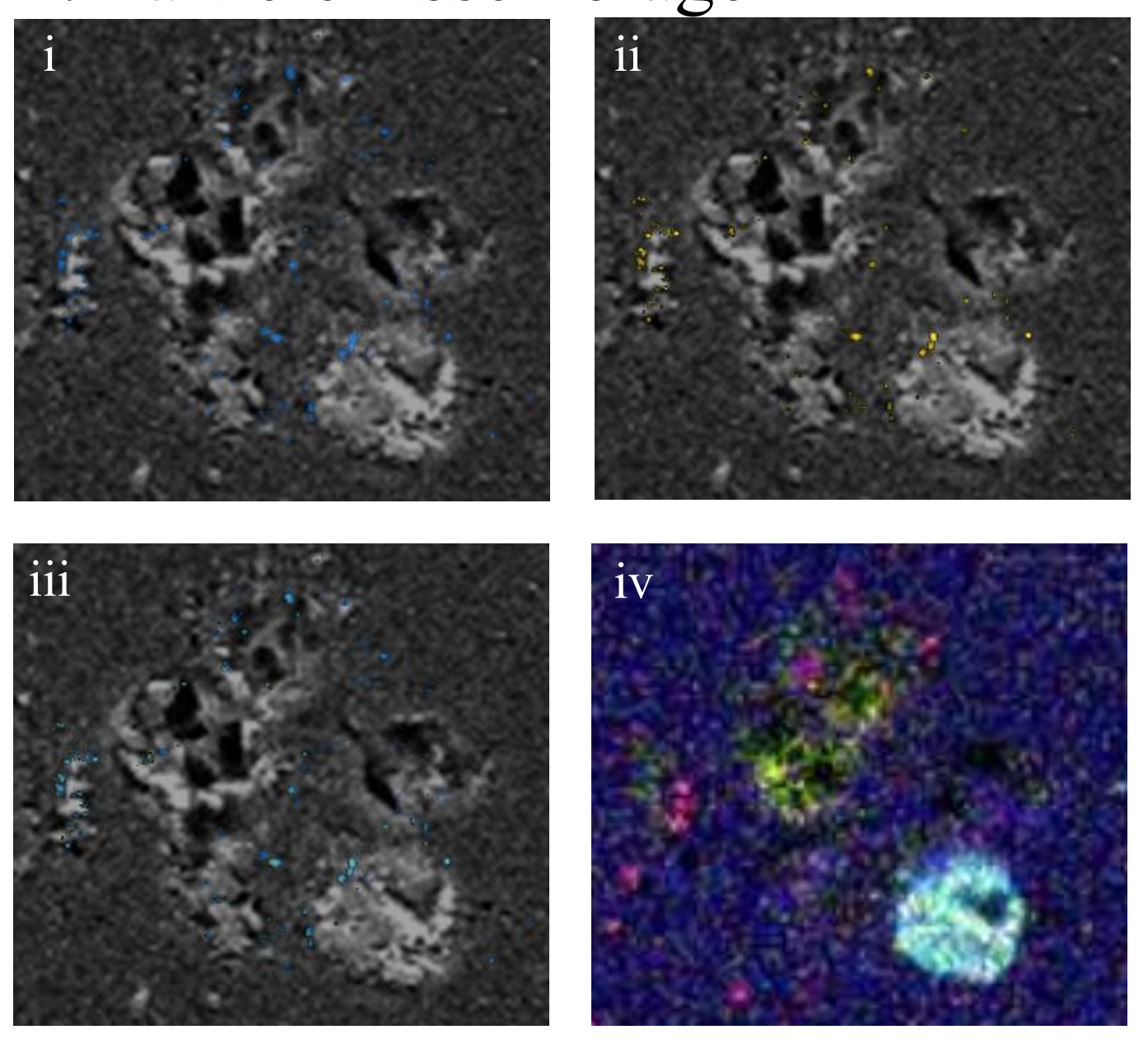

\section{3: Quartz}

4: Plagioclase / Orthoclase
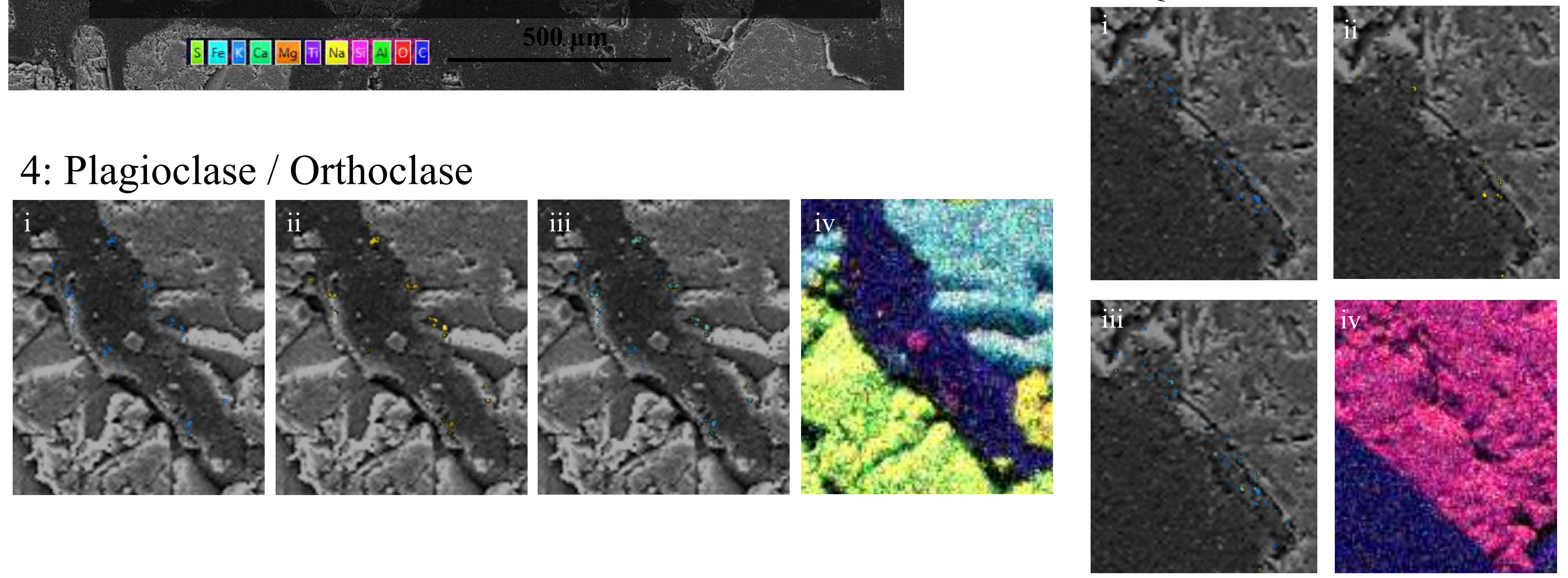
1 bioRxiv preprint doi: https:///doi.org/10.1101/2020.08.03.234146; this version posted August 3, 2020. The copyright holder for this preprint
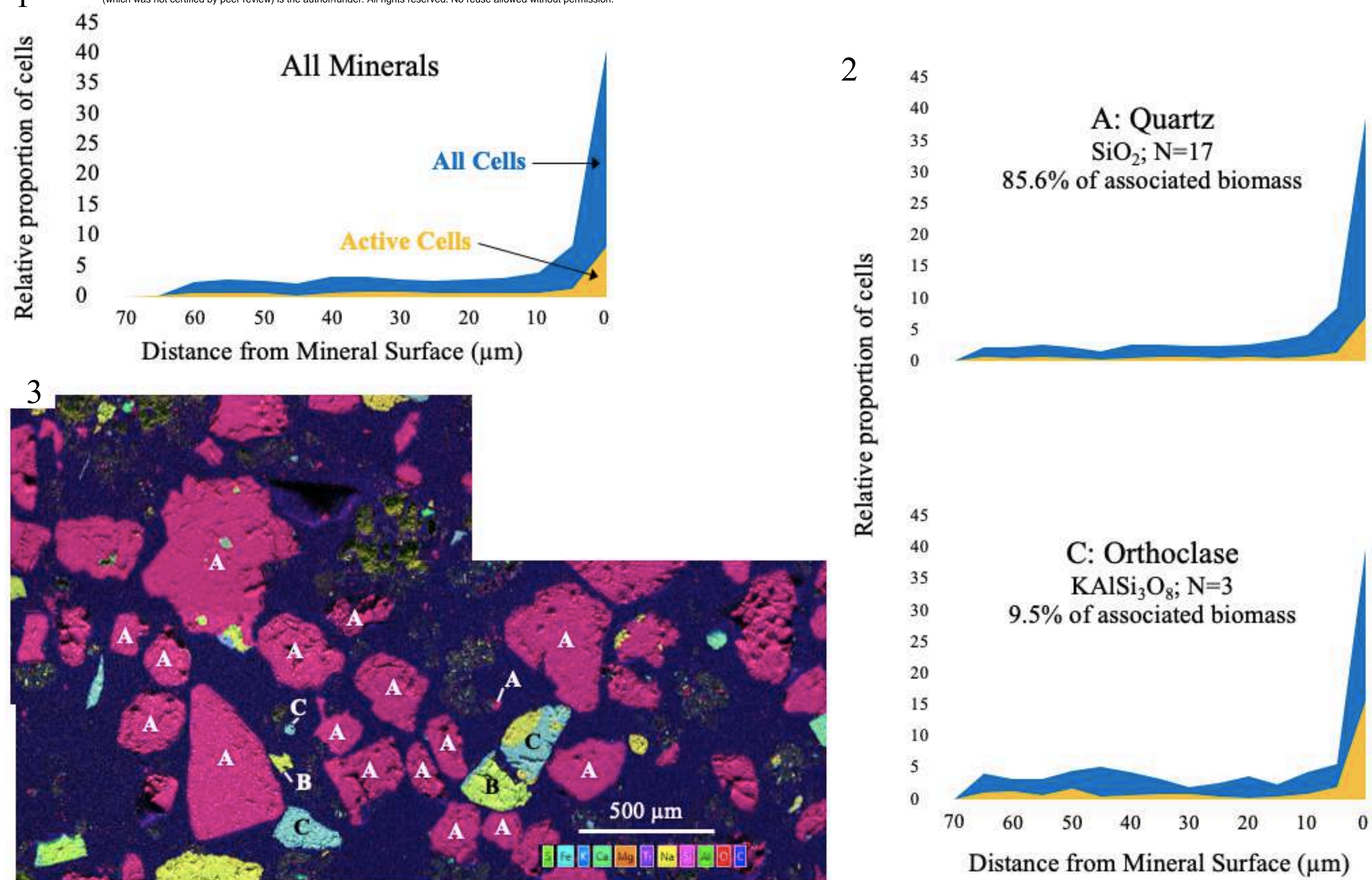


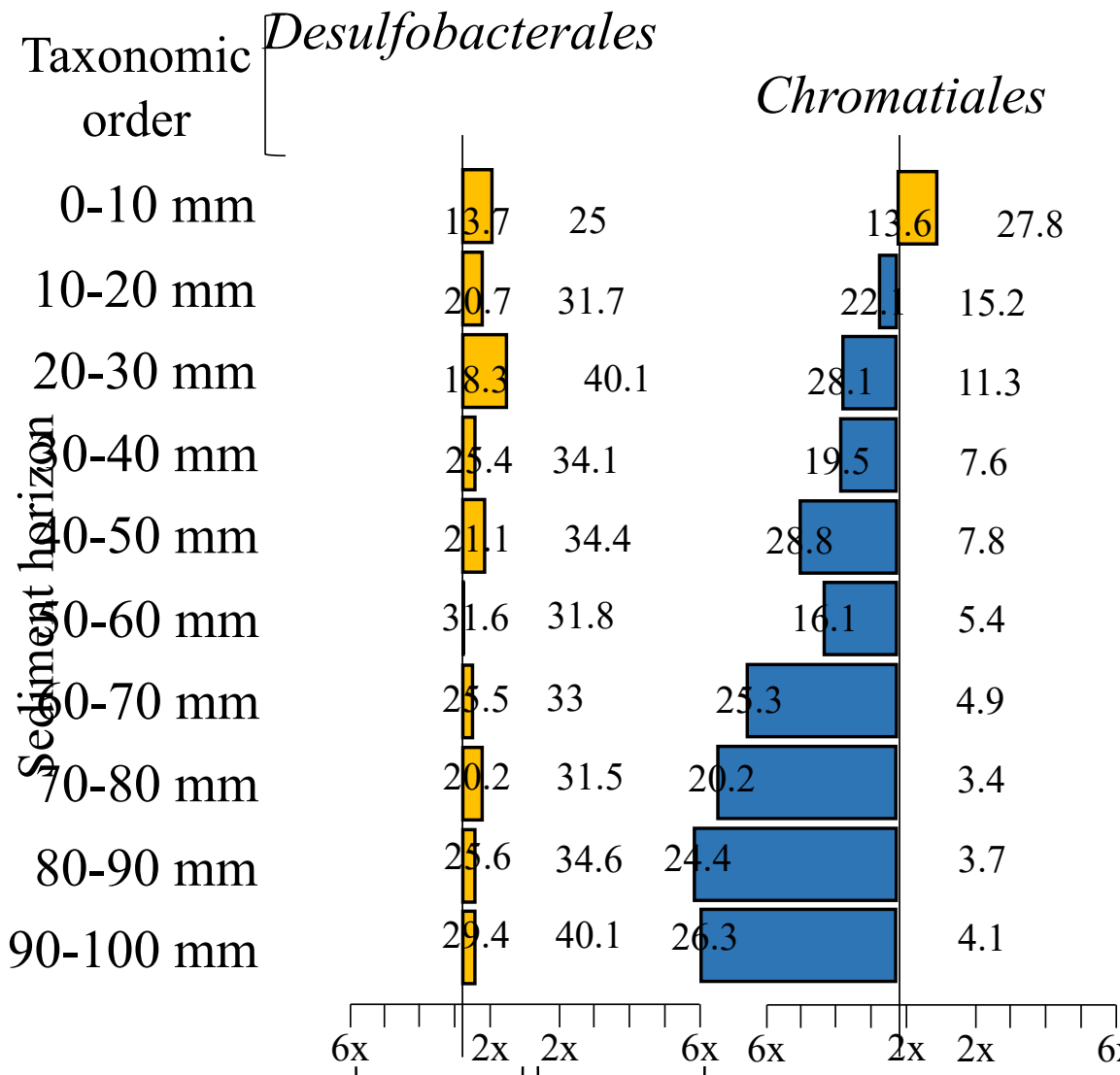
Fold-enrichment

Enriched in

Enriched in

inactive fraction

active fraction
Rhodobacterales
Betaproteobacteriales

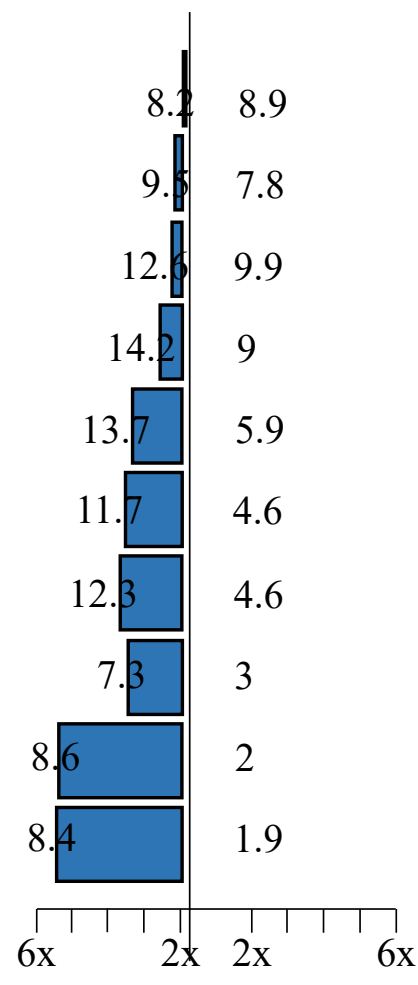

Desulfarculales

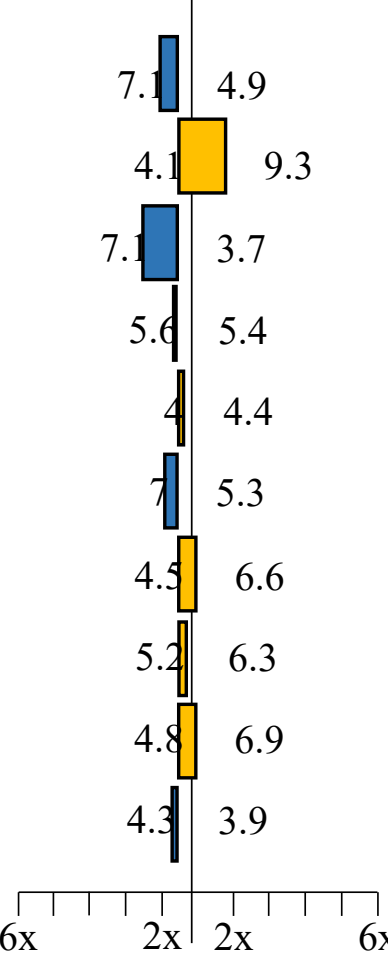

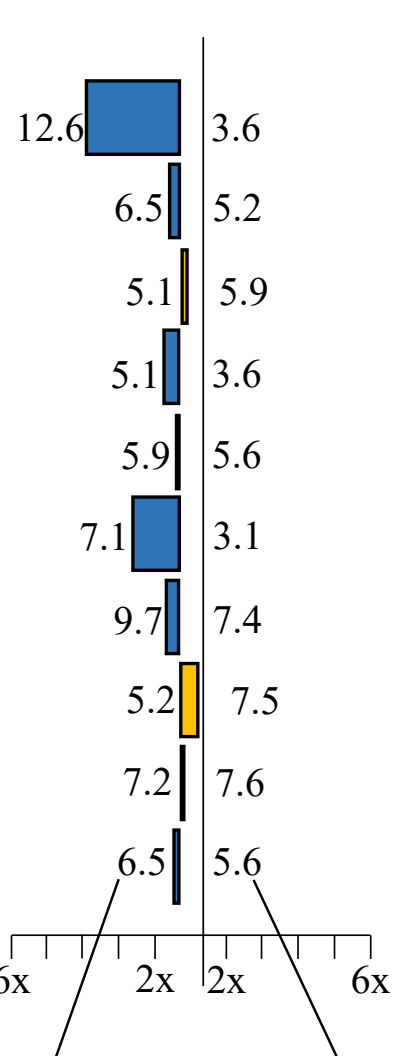

Sphingobacteriales

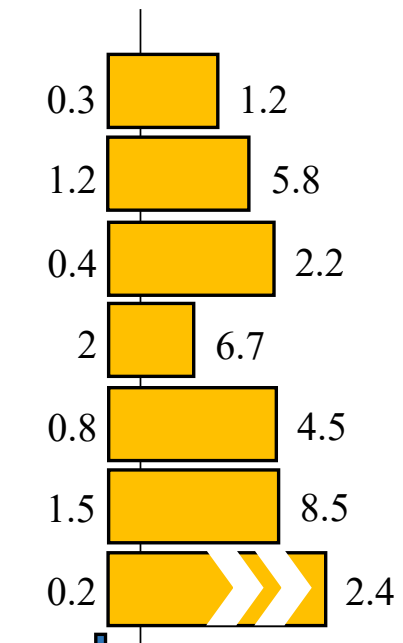

$3 \longdiv { 2 . 4 }$

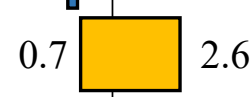

0.5

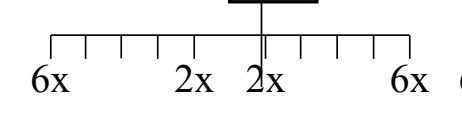

Cellvibrionales

\section{Pirellulales}

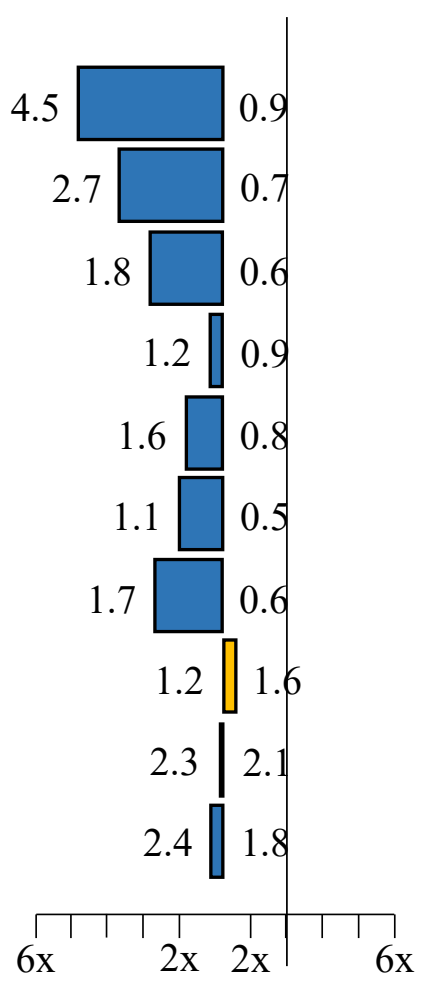

Inactive fraction relative abundance
Active fraction relative abundance

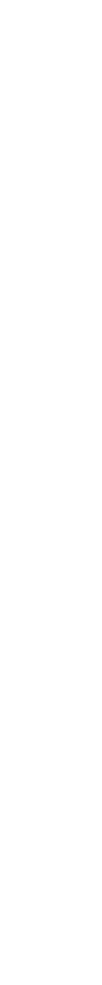




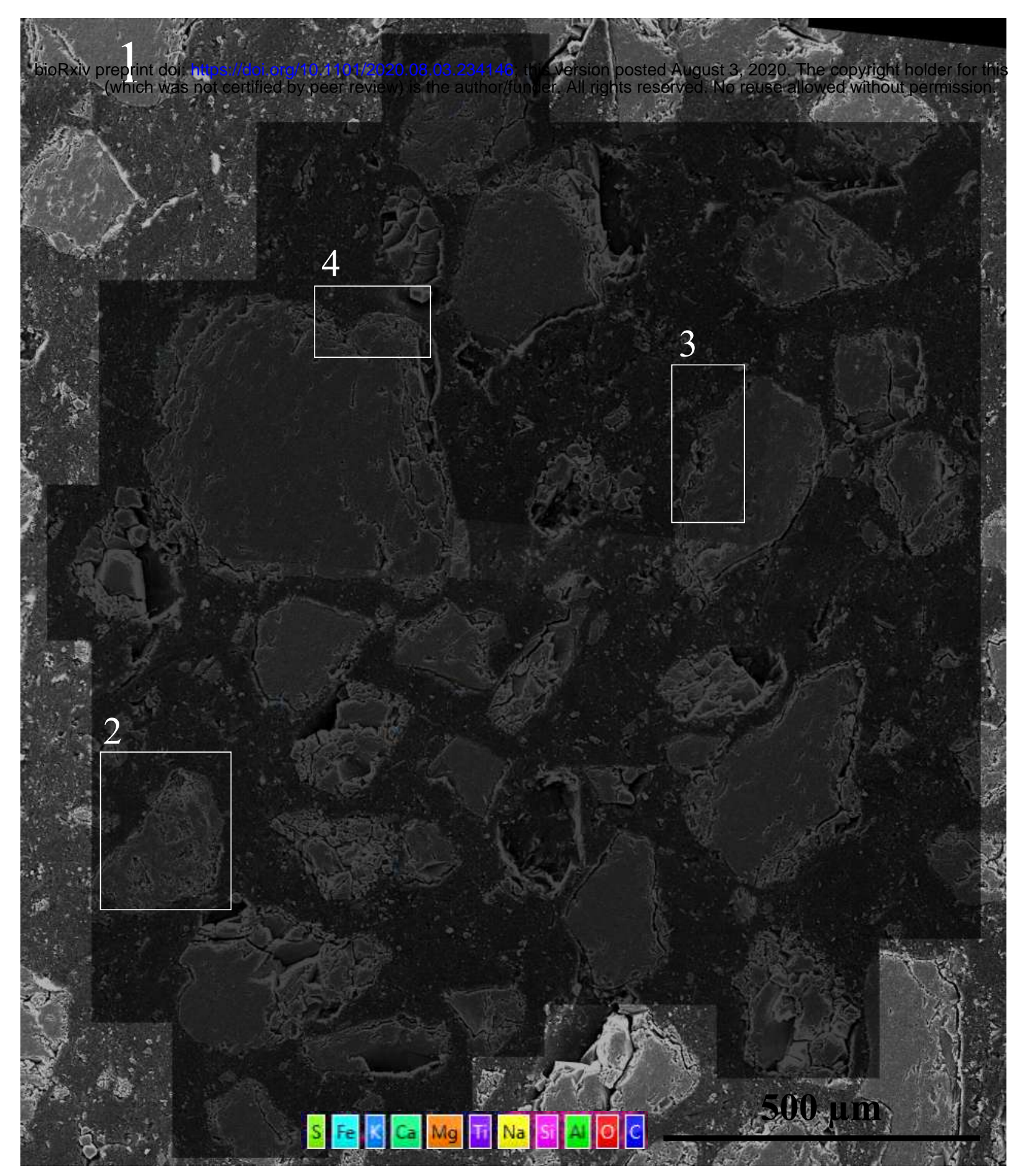

2: Plagioclase
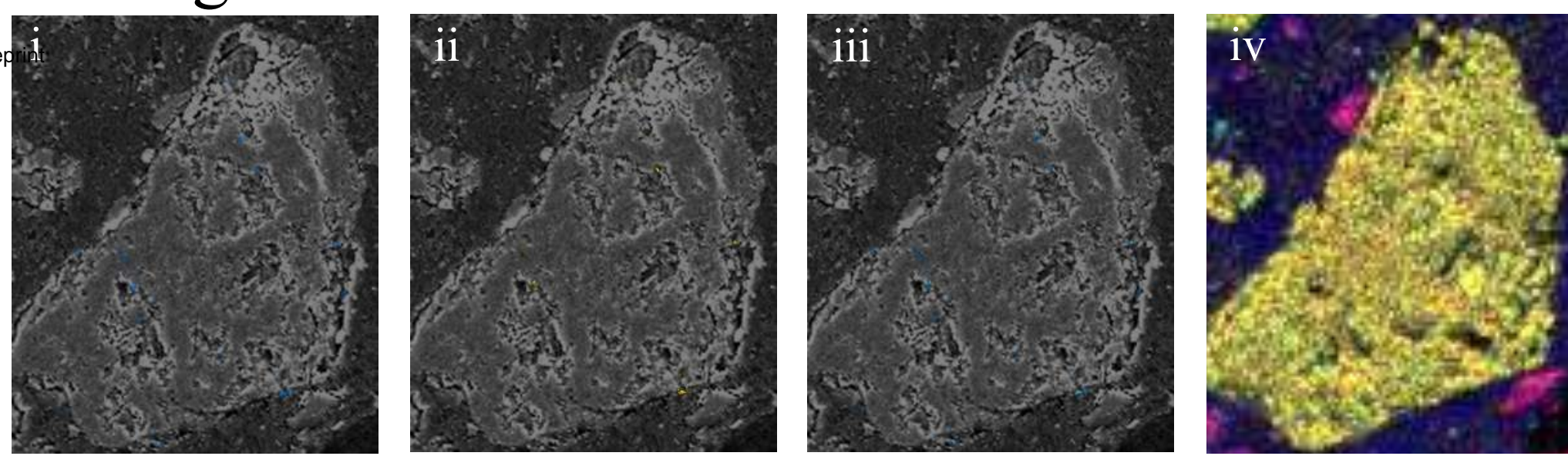

3: Albite / Orthoclase
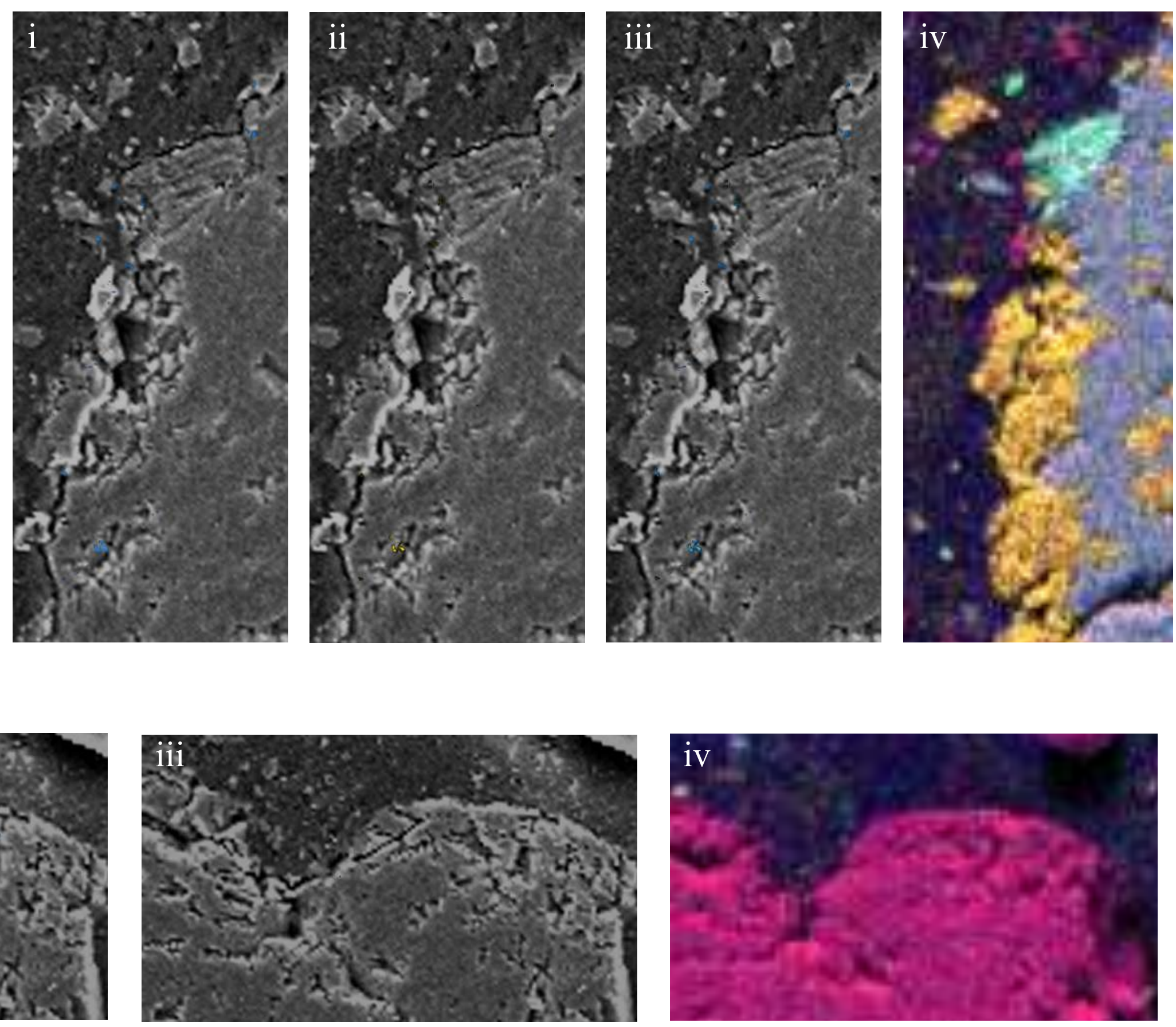

\section{4: Quartz}
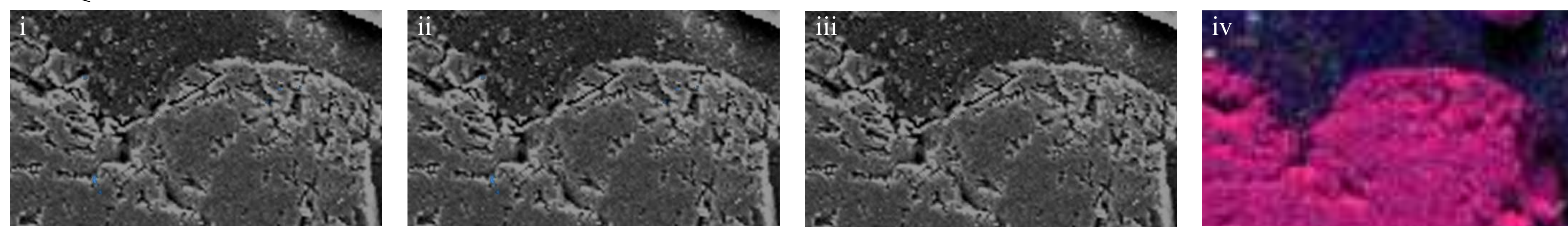


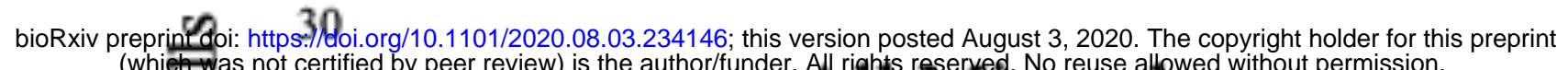

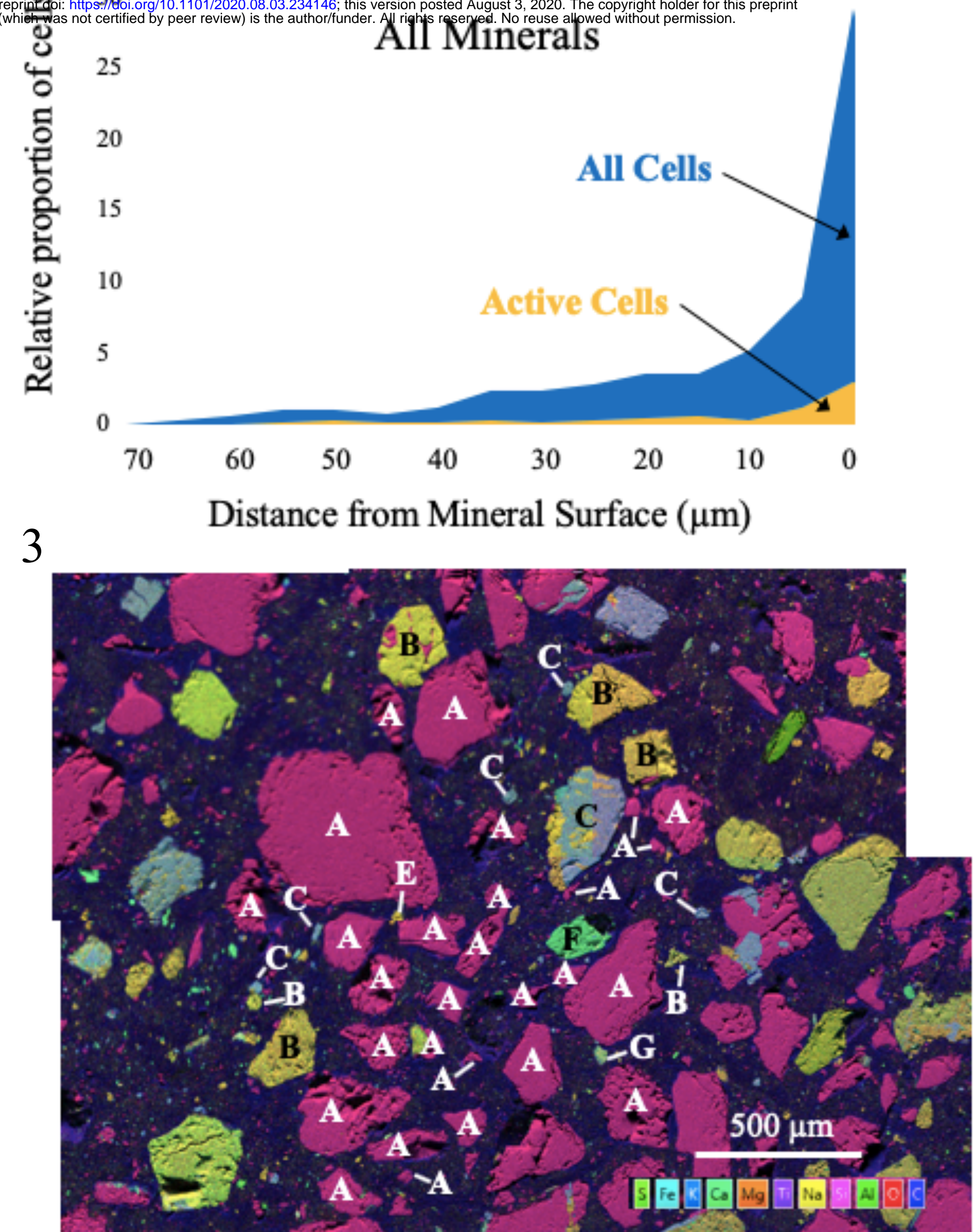

2

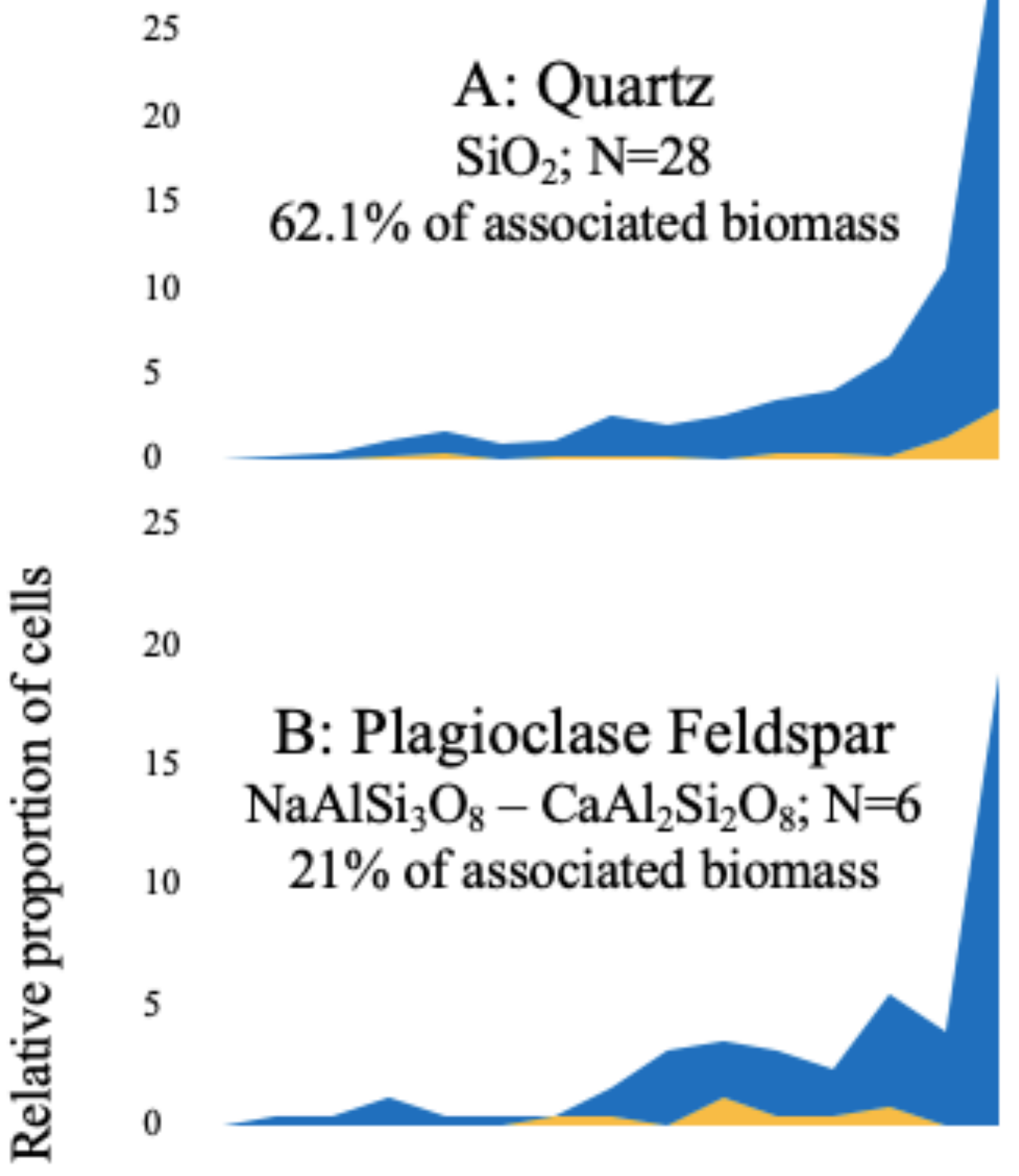

30

25

20

10

5

Distance from Mineral Surface $(\mu \mathrm{m})$ 


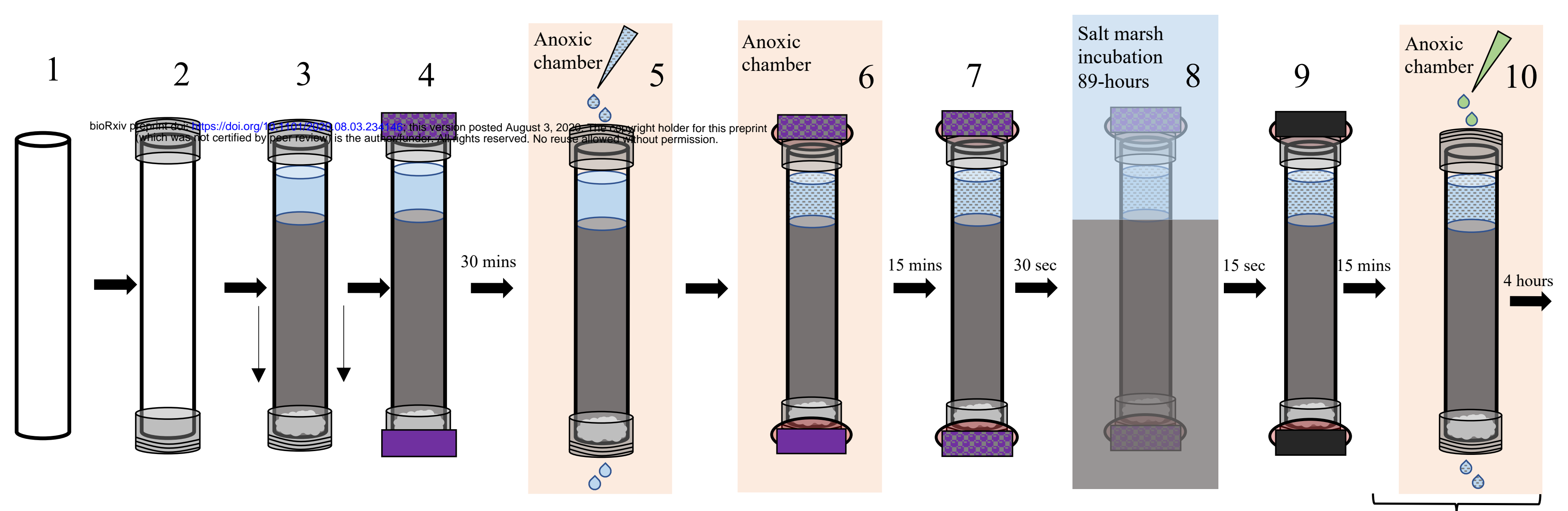

Correlative Microscopy Core

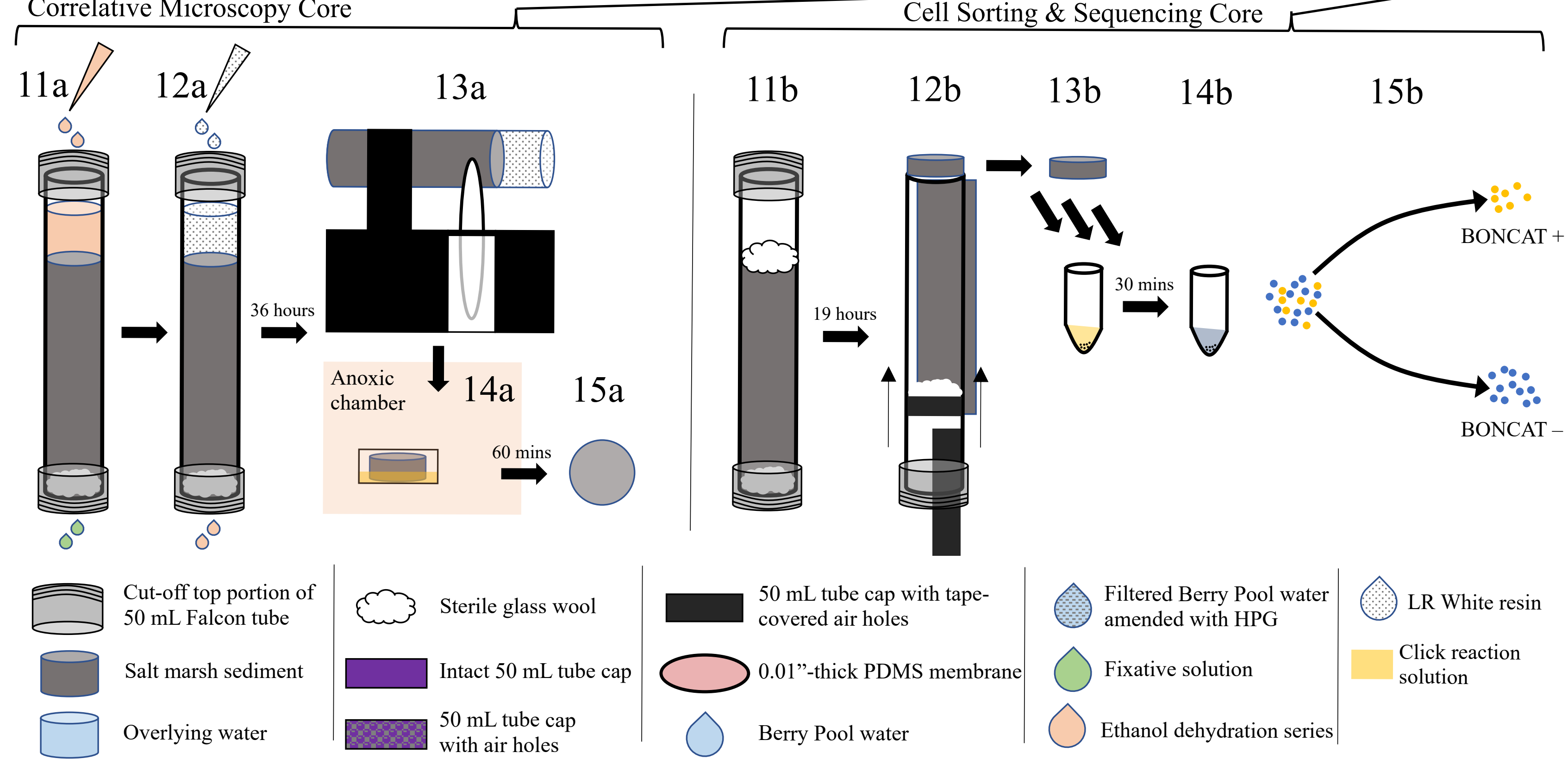


Table 1: Cell abundance and percentage of anabolically active cells as determined through fluorescence microscopy and BONCAT-FACS analyses.

\begin{tabular}{|r|r|r|r|r|}
\hline \multicolumn{3}{|c|}{ Fluorescence Microscopy Analysis } & \multicolumn{2}{|c|}{ FACS Analysis } \\
\hline Sediment Depth (mm) & Cell Abundance & $\begin{array}{r}\% \\
\text { Active }\end{array}$ & $\begin{array}{r}\% \\
\text { Active }\end{array}$ & Sediment Depth (mm) \\
\hline 7.6 & $1.95 \times 10^{9}$ & 51.3 & 70.0 & $0-10$ \\
\hline 12 & $2.86 \times 10^{9}$ & 22.3 & 22.4 & $10-20$ \\
\hline \multicolumn{3}{|c|}{} & 21.8 & $20-30$ \\
\cline { 4 - 5 } & & 10.5 & $30-40$ \\
\cline { 4 - 5 } & & 17.4 & $40-50$ \\
\hline
\end{tabular}

Where \% active values are available for both fluorescence microscopy and FACS datasets, the linear correlation coefficient was 0.99 . 
Table 2: Proportions of cells, and the anabolically active subsets, associated with mineral exteriors and interiors at the three horizons examined by correlative microscopy. For the biomass per surface area and volume, the relative proportion of biomass associated with a given mineral type was divided by the relative proportion of surface area or volume accounted for by that mineral type. Values less than 1 indicate fewer associated cells than would be expected given an even distribution of biomass across mineral perimeters or surfaces. Only mineral types that accounted for at least $5 \%$ of the observed biomass in a given horizon are included in this analysis.

\begin{tabular}{|c|c|c|c|c|c|c|c|c|c|}
\hline & & $\begin{array}{c}\% \text { of } \\
\text { Associated } \\
\text { Biomass }\end{array}$ & $\begin{array}{c}\% \\
\text { Active }\end{array}$ & $\begin{array}{c}\text { Associated } \\
\text { Biomass } \\
\text { per unit } \\
\text { Surface } \\
\text { Area }\end{array}$ & $\begin{array}{c}\text { Associated } \\
\text { Biomass } \\
\text { per unit } \\
\text { Volume }\end{array}$ & $\begin{array}{c}\% \\
\text { Outside }\end{array}$ & $\begin{array}{c}\% \text { of } \\
\text { Outside } \\
\text { Biomass } \\
\text { that was } \\
\text { Active }\end{array}$ & $\begin{array}{c}\% \\
\text { Inside }\end{array}$ & $\begin{array}{c}\% \text { of } \\
\text { Inside } \\
\text { Biomass } \\
\text { that was } \\
\text { Active }\end{array}$ \\
\hline \multirow{5}{*}{ 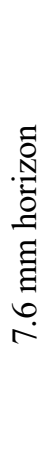 } & All Minerals & 100 & 51.3 & & & 77.5 & 51.7 & 22.5 & 52.8 \\
\hline & Quartz & 73.4 & 49.7 & 0.97 & 0.93 & 80.6 & 48.9 & 19.4 & 50.1 \\
\hline & Plagioclase & 10 & 62.6 & 1.3 & 1.44 & 75.9 & 63.6 & 24.1 & 59.5 \\
\hline & Orthoclase & 6.7 & 61.2 & 1.31 & 1.11 & 90 & 60.2 & 10 & 64.9 \\
\hline & Rutile & 5.3 & 66 & 1.26 & 1.5 & 42 & 78 & 58 & 57.3 \\
\hline \multirow{3}{*}{ 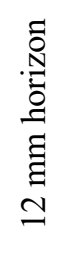 } & All Minerals & 100 & 22.3 & & & 80.2 & 20 & 19.8 & 24.8 \\
\hline & Quartz & 85.6 & 21.7 & 0.99 & 0.94 & 79.1 & 19.1 & 20.9 & 24.3 \\
\hline & Orthoclase & 9.5 & 29.8 & 1.24 & 1.68 & 89.6 & 30 & 10.4 & 29.8 \\
\hline \multirow{4}{*}{ 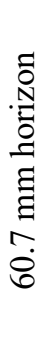 } & All Minerals & 100 & 12.1 & & & 62.2 & 10.3 & 37.8 & 14.9 \\
\hline & Quartz & 62.1 & 10.3 & 0.86 & 0.82 & 69.5 & 9.3 & 30.5 & 12.5 \\
\hline & Plagioclase & 21 & 12 & 1.52 & 1.6 & 45.6 & 7.6 & 54.4 & 15.6 \\
\hline & Orthoclase & 13.5 & 15 & 1.39 & 1.6 & 55.7 & 12.9 & 44.3 & 17.6 \\
\hline
\end{tabular}

